\title{
ACCRETION-INHIBITED STAR FORMATION IN THE WARM MOLECULAR DISK OF THE GREEN-VALLEY ELLIPTICAL GALAXY NGC 3226?
}

\author{
P. N. Appleton ${ }^{1}$, C. Mundell ${ }^{2}$, T. Bitsakis ${ }^{1,3}$, M. Lacy $^{4}$, K. Alatalo ${ }^{1}$, L. Armus ${ }^{5}$, V. Charmandaris ${ }^{6,7,8}$, \\ P.-A. DuC ${ }^{9}$, U. LisENFELD ${ }^{10}$, AND P. OGLE ${ }^{11}$ \\ ${ }^{1}$ NASA Herschel Science Center, Infrared Processing and Analysis Center, Caltech, 770S Wilson Avenue, Pasadena, CA 91125, USA; apple@ ipac.caltech.edu \\ ${ }^{2}$ Astrophysics Research Institute, John Moores University, Liverpool Science Park, 146 Brownlow Hill, Liverpool L3 5RF, UK \\ ${ }^{3}$ Instituto de Astronomia, National Autonomous University of Mexico, PO 70-264, 04510 Mexico D.F., Mexico \\ ${ }^{4}$ NRAO, Charlottesville, VA, USA \\ ${ }^{5}$ Spitzer NASA Herschel Science Center, 1200 East California Boulevard, Caltech, Pasadena, CA 91125, USA \\ ${ }^{6}$ Department of Physics, University of Crete, GR-71003 Heraklion, Greece \\ ${ }^{7}$ Institute for Astronomy, Astrophysics, Space Applications \& Remote Sensing, National Observatory of Athens, GR-15236 Penteli, Greece \\ ${ }^{8}$ Chercheur Associé, Observatoire de Paris, F-75014, Paris, France \\ ${ }^{9}$ Service d'Astrophysique, Laboratoire AIM, CEA-Saclay, Orme des Merisiers, Bat 709, F-91191 Gif sur Yvette, France \\ ${ }^{10}$ Dept. Fisica Teorica y del Cosmos, University of Granada, Edifica Mecenas, Granada, Spain \\ ${ }^{11}$ NASA Extragalactic Database, IPAC, Caltech, 1200 East California Boulevard, Caltech, Pasadena, CA 91125, USA \\ Received 2014 June 17; accepted 2014 October 26; published 2014 December 5
}

\begin{abstract}
We present archival Spitzer photometry and spectroscopy and Herschel photometry of the peculiar "Green Valley" elliptical galaxy NGC 3226. The galaxy, which contains a low-luminosity active galactic nucleus (AGN), forms a pair with NGC 3227 and is shown to lie in a complex web of stellar and $\mathrm{H}$ I filaments. Imaging at 8 and $16 \mu \mathrm{m}$ reveals a curved plume structure $3 \mathrm{kpc}$ in extent, embedded within the core of the galaxy and coincident with the termination of a $30 \mathrm{kpc}$ long $\mathrm{H}$ I tail. In situ star formation associated with the infrared (IR) plume is identified from narrowband Hubble Space Telescope (HST) imaging. The end of the IR plume coincides with a warm molecular hydrogen disk and dusty ring containing $0.7-1.1 \times 10^{7} M_{\odot}$ detected within the central kiloparsec. Sensitive upper limits to the detection of cold molecular gas may indicate that a large fraction of the $\mathrm{H}_{2}$ is in a warm state. Photometry derived from the ultraviolet (UV) to the far-IR shows evidence for a low star-formation rate of $\sim 0.04 M_{\odot} \mathrm{yr}^{-1}$ averaged over the last 100 Myr. A mid-IR component to the spectral energy distribution (SED) contributes $\sim 20 \%$ of the IR luminosity of the galaxy, and is consistent with emission associated with the AGN. The current measured star formation rate is insufficient to explain NGC 3226's global UV-optical "green" colors via the resurgence of star formation in a "red and dead" galaxy. This form of "cold accretion" from a tidal stream would appear to be an inefficient way to rejuvenate early-type galaxies and may actually inhibit star formation.
\end{abstract}

Key words: galaxies: active - galaxies: elliptical and lenticular, cD - galaxies: individual (Arp 94, NGC 3226)

Online-only material: color figures

\section{INTRODUCTION}

A complete picture of the evolution of galaxies and how they are grouped into two major branches seen in the local universe, namely the red sequence and the blue cloud (Strateva et al. 2001; Blanton et al. 2003; Hogg et al. 2004; Faber et al. 2007), is still a poorly understood problem in astronomy. One particular class of galaxy that may be relevant to the discussion of how stellar populations are built up over time are galaxies that fall in the so-called green valley, defined in terms of the ultraviolet (UV)-optical color lying in the range of $3<\mathrm{NUV}-r<5$, a color that places galaxies between the two main color classes. It has been suggested that galaxies in this region of color space can be characterized by a flow of star formation-quenched galaxies that migrate from the blue to the red (Martin et al. 2007) as star formation shuts down. However, the green valley galaxies may not be representative of a single transitional population (Westoby et al. 2007; Schawinski et al. 2014), but instead may be complicated by the availability of gas supply and the form of the quenching event that signals the start of a transition from blue to red. Active galactic nuclei (AGNs) may play a role in quenching.

Alternatively, galaxies can potentially move into the green valley (see Thilker et al. 2010) from the red sequence by a resurgence of star formation through accretion of gas from their environments: either through a gas-rich minor merger or through acquisition of tidal debris. In this paper, we present evidence of a possible ongoing $\mathrm{H}$ I gas accretion event in the center of the elliptical galaxy NGC 3226 and explore its implications for this kind of color change.

The green valley (NUV $-r=4.35$; this paper) elliptical galaxy NGC 3226 is a companion of the large, disturbed, barredspiral galaxy NGC 3227 forming the Arp 94 system (Figure 1). The core of NGC 3226 contains a dusty disk or partial ring (see Figure 1 inset), and hints of its LINER-like optical spectra can be traced back to Rubin \& Ford (1968). It was spectroscopically classified as a LINER type 1.9 by Ho et al. (1997), and it is generally accepted as a classic example of a low-luminosity, $\mathrm{X}$-ray-bright AGN, most likely the result of radiatively inefficient accretion onto a supermassive black hole (Ho \& Ulvestad 2001; George et al. 2001; Ho et al. 2009; Liu \& Wu 2013 and references therein).

Evidence that NGC 3226 and NGC 3227 are interacting was first presented by Rubin \& Ford (1968) through optical spectroscopy. Subsequent Very Large Array (VLA) observations revealed $100 \mathrm{kpc}$ scale plumes of neutral hydrogen extending over 20 arcmin to the north and south of the galaxy pair (Mundell et al. 1995), shown in Figure 2 (top panel), which are most likely tidally generated structures. 


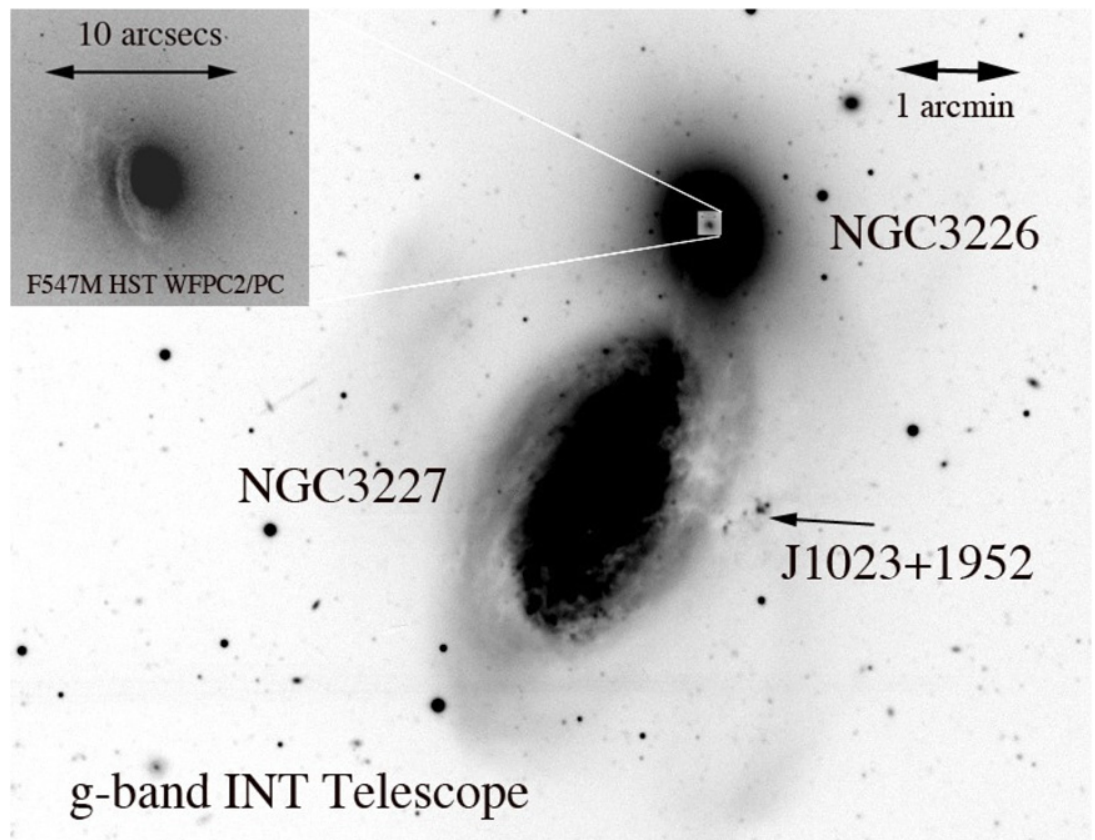

Figure 1. $g$-band image of the Arp 94 system. NGC 3226 lies to the north of NGC 3227 and the H I-rich dwarf galaxy J1023+1952 to the west. The inset shows a $V$-band HST image of the core of NGC 3226 that contains a partial dust ring or disk.

A contrast-enhanced MegaCam image from Duc et al. (2014), Figure 2 (bottom panel), exhibits a complex set of faint stellar arcs and ripples around both NGC 3227 and NGC 3226 (like those described by Schweizer \& Seitzer 1988). This suggests a rich dynamical history for the system. NGC 3226 shows several huge loops and a narrow optical filament extending from the galaxy to the northeast at a position angle of $\sim 30 \mathrm{deg}$. The visible light imagery presents a complexity that is hard to reconcile with a single tidal interaction between NGC 3227 and NGC 3226. Rather, the structures around NGC 3226 imply that this galaxy is itself the remnant of a recent merger that has launched stellar debris into the joint potential of what was probably a system of at least three constituent galaxies.

The H I map shown in Figure 2 seems consistent with two long tidal tails (north and south) perhaps associated with NGC 3227. However, this may be an oversimplification. First, the system is complicated by the discovery of a 40 arcsec scale HI-rich dwarf galaxy (J1023+1952) located in front of the western disk of NGC 3227 (Mundell et al. 1995). This galaxy is physically distinct from NGC 3227, shows independent rotation (Mundell et al. 2004), and appears to be experiencing a burst of star formation in part of its disk (see also Figure 1). Studying the molecular and optical-IR properties in more detail, Lisenfeld et al. (2008) suggested that J1023+1952 may be formed at the intersection of two stellar streams. Second, although the southern Hi tail contains an optical counterpart (seen faintly in Figure 2) connecting it to NGC 3227, the northern plume is less obviously correlated with the faint visible light structures and is most likely kinematically associated with NGC 3226 not NGC 3227.

We present Spitzer, Herschel, and Hubble observations of NGC 3226, revealing the existence of a warm molecular disk in its core, which may be fed from a dusty filament that is likely tidal debris falling back onto NGC 3226. NGC 3226 has a heliocentric velocity of $1313 \pm 16 \mathrm{~km} \mathrm{~s}^{-1}$ (Simien \& Prugniel 2002), and we adopt a distance of $15.1 \mathrm{Mpc}$ based on a heliocentric velocity of $1135 \mathrm{~km} \mathrm{~s}^{-1}$ for NGC 3227 (the larger member of the pair) and $H_{0}=75 \mathrm{~km} \mathrm{~s}^{-1} \mathrm{Mpc}^{-1}$.

\section{THE OBSERVATIONS}

Table 1 summarizes the archival observations used in this paper, including Spitzer mid-infrared imaging and spectroscopy, Herschel far-infrared (IR) photometric mapping, and Hubble Space Telescope imaging.

\subsection{Spitzer Observations}

Some of the mid-IR observations described in this paper were made shortly after the launch of Spitzer, during the instrument verification phase of the mission. IRAC (Fazio et al. 2004) imaging in all four bands (3.6, 4.5, 6 and $8 \mu \mathrm{m})$ was performed using a seven-point dither pattern to encompass both NGC 3227 and NGC 3226, although the emphasis here is on these data for NGC 3226 . Observations using the small-map photometry mode of the MIPS (Rieke et al. 2004) instrument were made at $24 \mu \mathrm{m}$. Infrared Spectrograph (IRS, Houck et al. 2004) photometric mapping using the blue peak-up camera provided a $16 \mu \mathrm{m}$ image of the galaxy as part of the first calibration observations of this mode. Raw data from these latter observations were processed through the Spitzer Science Center standard S18 pipelines, and the 16 and $24 \mu \mathrm{m}$ maps were further processed using the MOPEX mapping package (Makovoz \& Marleau 2005).

A small spectral map was taken in the IRS Short-Low (SL) module, covering the wavelength range of 5-15 $\mu \mathrm{m}$. A $9 \times 1$ grid of spectra was obtained, centered on the nucleus of NGC 3226: each single spectrum covered an area $57 \times 3.7 \operatorname{arcsec}^{2}$. At each slit position, a spectrum was taken and the slit was moved 2.5 arcseconds (two-thirds of a slit width) before taking the next exposure. Reduction of the spectra were performed using the Spitzer Science Center (SSC) IRS S18 pipeline and resulted in two-dimensional (2D) images of the spectral orders. The spectral cubes were further processed using the CUBISM package (Smith et al. 2007a). Local sky subtraction was performed in the construction of the spectral cubes using off-target observations of identical integration time. Spectral line fluxes were extracted using PAHFIT (Smith et al. 2007b). 

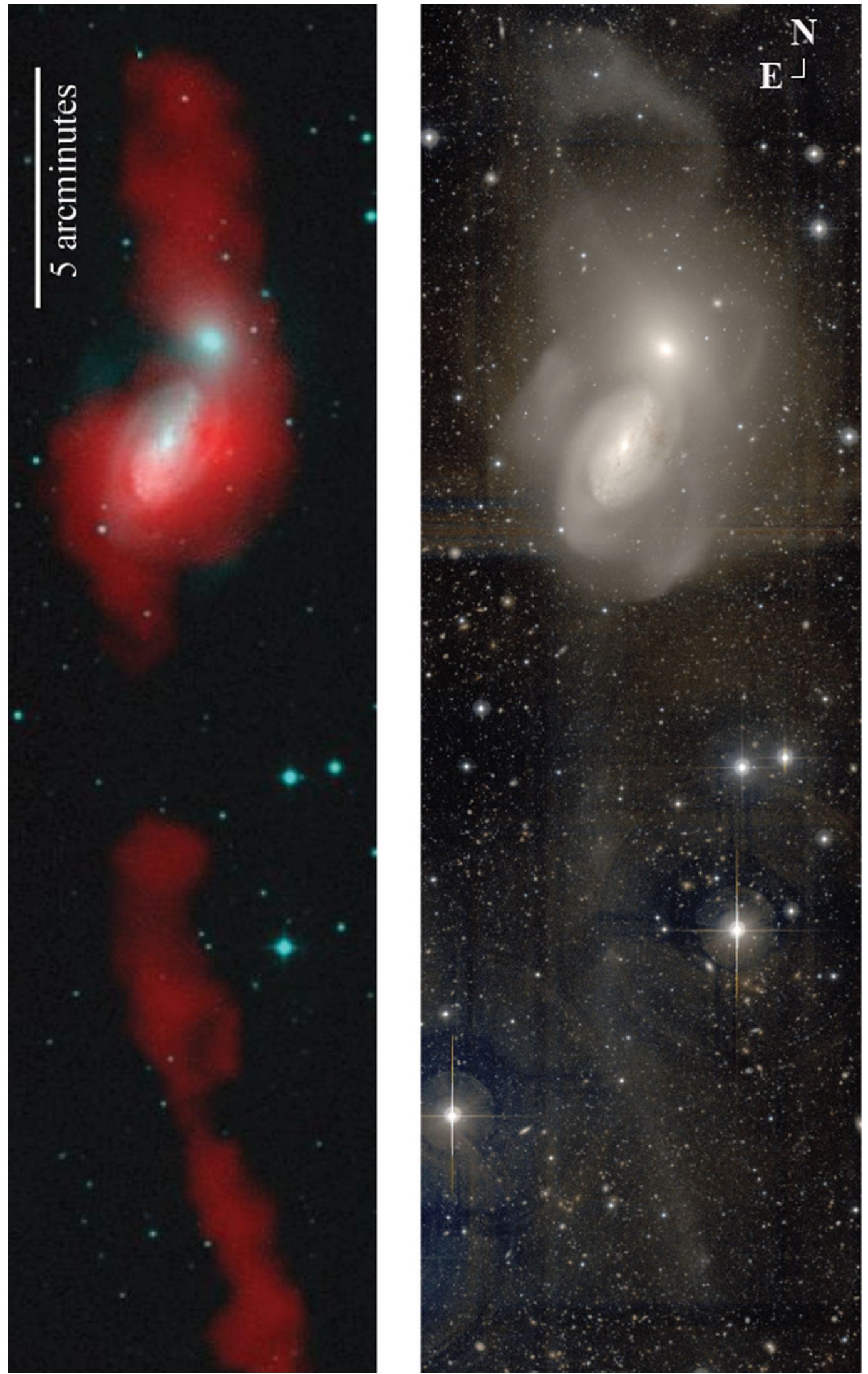

Figure 2. Top: the large-scale neutral hydrogen emission (red) superimposed on a deep optical image of the galaxy from observations by Mundell et al. (1995). The figure shows the large scale $(20 \mathrm{arcmin})$ of the Hi plumes, suggesting that the members of Arp 94 have interacted tidally in the past. Bottom: composite g+r MegaCam/CFHT image of the Arp94 system (Duc et al. 2014) rendered with an "arcsinh stretch" to bring out the faint structure. The image, matched in scale to the H I image, shows evidence of considerable tidal debris, including shells and ripples. The southern H I plume appears to have a faint stellar counterpart whereas the northern filament is less correlated with the faint optical light.

(A color version of this figure is available in the online journal.) 
Table 1

Journal of Spitzer and Herschel Observations

\begin{tabular}{|c|c|c|c|c|}
\hline Instrument & UT Date & Instrument Mode & $\begin{array}{l}\text { Integration Time } \\
\text { on Source }(\mathrm{s})\end{array}$ & $\begin{array}{l}\text { Wavelength } \\
\qquad(\mu \mathrm{m})\end{array}$ \\
\hline Spitzer IRAC ${ }^{\mathrm{a}}$ & $11: 23: 2003$ & 7-pt. med. dither & 288 & $3.6,4.5,6.8,8.0$ \\
\hline Spitzer MIPS ${ }^{\mathrm{a}}$ & 11:24:2003 & Small-map photometry & 300 & 24 \\
\hline Spitzer IRS ${ }^{\mathrm{b}}$ & $12: 17: 2003$ & Peak-up imaging map & 60 & 16 \\
\hline Spitzer IRS ${ }^{\mathrm{c}}$ & $11: 28: 2003$ & Short-low $9 \times 1$ map & 60 & $5-14$ \\
\hline Spitzer IRS ${ }^{\mathrm{d}}$ & 05:26:2005 & Short-Hi/Long-Hi staring & $365 / 487$ & $9.7-37.2$ \\
\hline Herschel ${ }^{\mathrm{e}}$ PACS & $15: 16: 2011$ & Photometry Scan Map & 600 & 70 and 160 \\
\hline Herschel $^{\mathrm{f}}$ SPIRE & 05:30:2010 & Photometry Large map & 1112 & 250,350 and 500 \\
\hline Hubble ${ }^{\mathrm{g}} \mathrm{ACS}$ & 03:08:2003 & F814W filter & 700 & $0.8060,0.6583 .9$ \\
\hline Hubble ${ }^{\mathrm{h}}$ WFPC2/PC & 03:18:1997 & F547M filter & 460 & 0.547 \\
\hline
\end{tabular}

Notes.

a Observations from P. N. Appleton; Program ID = 1054.

b "In-orbit check-out" observations from J. Houck; Program ID $=668$.

c "In-orbit check-out" observations from J. Houck; Program ID $=1401$.

${ }^{\mathrm{d}}$ Archival observations from C. Leitherer; Program ID $=3674$.

${ }^{\mathrm{e}}$ Archival observations from GT1_lspinogl_2; obsids $=1342221146$.

${ }^{\mathrm{f}}$ Archival observation from KPOT_seales01_1; obsid =1342197318.

g Archival observations from GTO/ACS program 9293; PI: H. Ford.

${ }^{\mathrm{h}}$ Archival observation from WFPC2/PC program 6837; PI: L. Ho.

Spitzer high-resolution (long-high $(\mathrm{LH})=18.7-37.2 \mu \mathrm{m}$ and short-high $(\mathrm{SH})=9.9-19.6 \mu$ m modes $)$ spectra taken at the center of NGC 3226 (see Table 1) were processed through the SSC S18 pipeline. The $\mathrm{LH}$ and SH apertures subtend an area of $22.3 \times 11.1 \operatorname{arcsec}^{2}$ and $11.3 \times 4.7 \operatorname{arcsec}^{2}$, respectively. The two dithered spectra taken in each order were blinked against one another to allow identification of cosmic-ray glitches and "rogue" pixels, whose values were then replaced by interpolation from surrounding pixels. Spectral extractions were made using the SSC software package SPICE (Spitzer IRS Custom Extraction-a Java-based interactive analysis tool), and line fluxes were extracted using the ISAP package (Sturm et al. 1998).

\subsection{Herschel Observations}

Herschel ${ }^{12}$ observations at 70 and $160 \mu \mathrm{m}$ were obtained with the PACS photometer (Poglitsch et al. 2010), and at 250, 350, and $500 \mu \mathrm{m}$ with the SPIRE (Griffin et al. 2010) photometer. Raw data, Level 0, for both instruments were de-archived from the Herschel Science Archive and processed to Level 1 using the HIPE (Herschel Interactive Processing Environment) 11.1 software (Ott 2006). For PACS, map-making beyond Level 1 was performed using the "Scanamorphos" software package (Roussel 2013). These PACS observations combined scan and cross-scan maps covering $8.3 \times 8.3 \mathrm{arcmin}^{2}$ and were obtained at medium speed, resulting in a total on-source integration times of 600 s. SPIRE imaging (Griffin et al. 2010) was performed in large map mode covering an $8 \times 8$ arcmin area centered on the NGC 3226/7 pair. SPIRE data were processed through the "Destripper" pipeline in HIPE 11. Details of the observing programs and other information is provided in Table 1.

\subsection{Hubble Space Telescope Observations}

Hubble Space Telescope (HST $\mathrm{H} \alpha$ observations (originally discussed by Martel et al. (2004) in a different context) taken with the Advanced Camera for Surveys (ACS) were obtained of

12 Herschel is an ESA space observatory with science instruments provided by the European-led Principal Investigator consortia and with important participation from NASA (Pilbratt et al. 2010). the region surrounding NGC 3226 from the Hubble archive (see Table 1). The observations were obtained in the broadband F814W continuum filter, in which negligible contamination from emission lines is expected, and in a F658N narrowband filter, which includes the redshifted $\mathrm{H} \alpha_{\lambda 6563}$ and $[\mathrm{N} \text { II }]_{\lambda \lambda 6548,6584}$ emission lines. Data were processed with the on-the-flycalibration procedures on retrieval from the $H S T$ archive, and the final drizzled images were post-processed as follows. The two images were initially flux calibrated using their respective PHOTFLAM keywords to ensure correct continuum subtraction with the F814W and the optimal scaling was verified by ensuring that pure continuum sources, such as background galaxies, were correctly subtracted. The F814W was shifted slightly ( $\Delta x=-0.46$ pixels, $\Delta y=-0.37$ pixels) for correct alignment with the F658N image. A revised equivalent PHOTFLAM for the continuum-subtracted F658N image was then computed using CALCPHOT in the IRAF SYNPHOT package. Although the F658N bandpass includes contamination from the [N II] emission lines, their contribution is assumed to be no more than $\sim 20 \%$ in normal star forming regions. ${ }^{13}$ We therefore followed Martel et al. (2004) and assumed a single $5 \AA$ A wide Gaussian line at the $\mathrm{H} \alpha$ wavelength appropriate for the redshift of NGC 3226. We also present archival $V$-band $H S T$ observations of the core of NGC 3226 obtained in 1997 with the WFPC2/PC.

\section{RESULTS}

\subsection{A Dusty Star Forming Filament Inside the Optical Dimensions of NGC 3226}

In Figure 3, we show IRAC images of NGC 3226. Although this elliptical galaxy shows only a smooth stellar distribution at $3.5 \mu \mathrm{m}$ and $4.5 \mu \mathrm{m}$, the galaxy contains a filament of emission in the $8 \mu \mathrm{m}$ band (and faintly in the $5.8 \mu \mathrm{m}$ band). The latter is centered close to the $7.7 \mu \mathrm{m}$ polycyclic aromatic hydrocarbon (PAH) complex, whereas the former may be from the $6.3 \mu \mathrm{m}$ PAH band. This structure (noticed by Tang et al. 2009; Lanz et al. 2013), lies well inside the $D_{25}$ optical dimensions of the galaxy $\left(3.16 \times 2.81 \operatorname{arcmin}^{2}\right.$ de Vaucouleurs et al. 1991) and is

\footnotetext{
13 If shocks were present, this approximation may not be appropriate and we
} may overestimate the $\mathrm{H} \alpha$ contribution. 


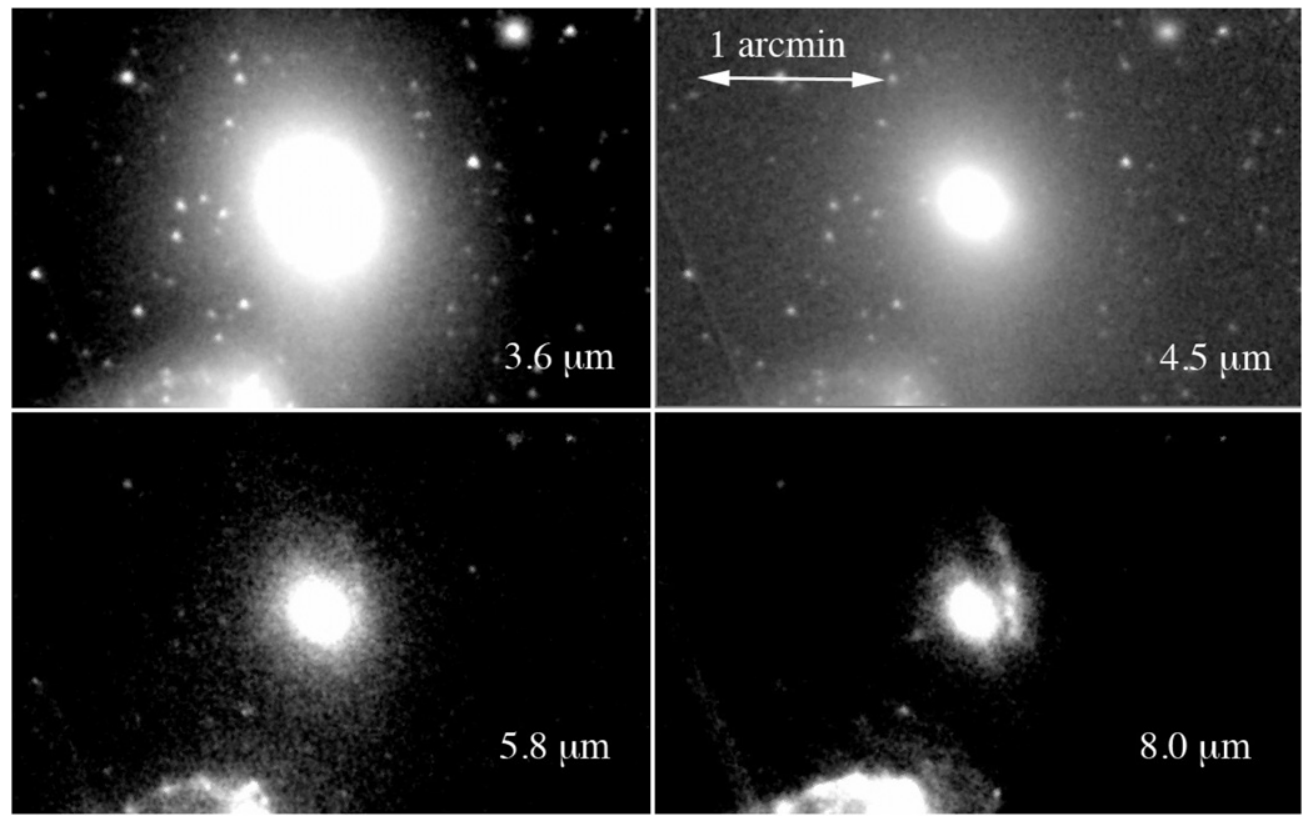

Figure 3. Grayscale representation of the 3.6, 4.5, 5.8, and $8.0 \mu \mathrm{m}$ Spitzer IRAC images of NGC 3226. North is to the top and east is to the left. Note the filament seen embedded within the galaxy in the $8 \mu \mathrm{m}$ image and more faintly at $5.8 \mu \mathrm{m}$.

composed of knots of emission extending northward from the western side of the inner galaxy. The filament's southernmost extent terminates close to a prominent dust lane seen in optical images of the galaxy (see Figure 1). We argue later that the feature is detected in $\mathrm{H} \mathrm{r}$ emission and represents the termination of a possible infalling stream of gas in a tidal tail.

Figure 4 shows the relationship between the IRAC $8 \mu \mathrm{m}$ emission and the continuum-free $H S T \mathrm{H} \alpha+[\mathrm{N}$ II] image at both full resolution and smoothed to show extended emission. H II region complexes are seen associated with the main $8 \mu \mathrm{m}$ clumps in the filament. Observed and derived properties from the photometry are presented in Table 2 for the knots labeled A-E in Figure 4 . We present the $8 \mu \mathrm{m}$ flux density and luminosity (Table 2; Columns 2 and 4) converted to a stellar-free flux density $\mathrm{S}(8)_{\text {dust }}$ by subtracting $25 \%$ of the $\mathrm{S}(3.6 \mu \mathrm{m})$ flux density from the same aperture from the $8 \mu \mathrm{m}$ emission to provide a "dust+PAH" band flux (Calzetti et al. 2007). The H $\alpha$ flux and luminosity are estimated from the $\mathrm{H} \alpha H S T$ image for each of the regions (uncorrected for extinction; Table 2, Columns 3 and 5).

Since the filament was not detected at $24 \mu \mathrm{m}$, we estimated the star formation rates in the filament regions using two methods. The first is based simply on the observed $\mathrm{H} \alpha$ luminosity $L$ $(\mathrm{H} \alpha)$ only (Kennicutt 1998; Table 2, Column 5), providing a star formation rate (SFR; H $\alpha$; Table 2, Column 7) assuming a Kroupa IMF. This SFR is uncorrected for extinction. The second method uses both the $8 \mu \mathrm{m} \operatorname{dust}(+\mathrm{PAH})$ luminosity and the observed $\mathrm{H} \alpha$ luminosity from the HST image and the empirical relationship of Kennicutt et al. (2009) between these quantities and the unobscured $\mathrm{H} \alpha$ corr luminosity (Table 2, Column 6). This provides an unobscured SFR (Table 2, Column 8). As expected, the first method yields slightly lower SFRs than the combined visible-IR method, most likely due to modest extinction in the filament. The SFRs in the filament clumps are very low, between $1-3 \times 10^{-3} M_{\odot} \mathrm{yr}^{-1}$.

Further insight into the IR properties of the filament can be obtained by looking at the observations of NGC 3226 at longer wavelengths. The filament is detected at $16 \mu \mathrm{m}$, but not $24 \mu \mathrm{m}$. Figure 5 shows the close correspondence between the $16 \mu \mathrm{m}$ emission (contours) and the $8 \mu \mathrm{m}$ image centered on the 7.7-8.6 PAH bands. Since no detectable emission is seen at $24 \mu \mathrm{m}$ (Figure 5, right panel), this implies that the $16 \mu \mathrm{m}$ image is detecting line emission, either narrow $17 \mu \mathrm{m}$ 0-0 S(1) molecular hydrogen emission, broader $16 \mu \mathrm{m}$ PAH emission, or both. It is therefore likely that most of the light in the IR originates in line emission rather than warm dust.

Emission from PAHs would be expected from the previous discussion on star formation, as the PAHs would be thermally spiked by UV radiation. Since our IRS spectroscopic observations did not cover this region, we cannot conclusively prove whether either large composite planar-bending mode PAHs are responsible for the $16 \mu \mathrm{m}$ emission or warm molecular hydrogen. Such emission might be expected if the filament contains shocks.

Figure 6 shows Herschel PACS and SPIRE images of the galaxy compared with an optical image on the same scale. The $70 \mu \mathrm{m}$ map, which has similar spatial resolution to the Spitzer $24 \mu \mathrm{m}$ image of Figure 5 (right panel), shows an unresolved core (on a scale of $<5.5$ arcsec $=400 \mathrm{pc}$ ) with a faint extended (35 arcsec $=2.5 \mathrm{kpc}$ ) component elongated roughly along a position angle of $\sim 42^{\circ}-45^{\circ}$ (north through east), especially to the north.

The filament may contain colder dust as it is weakly detected in the PACS $160 \mu \mathrm{m}$ image to the northwest of the dusty core and is possibily detected in the lower-resolution SPIRE images (Figure 6).

\subsubsection{A Neutral Hydrogen Counterpart to the IRAC Dust Filament}

In Figure 7, we show a series of channel-map images showing $\mathrm{H}_{\mathrm{I}}$ column density, in steps of $41 \mathrm{~km} \mathrm{~s}^{-1}$, obtained by Mundell et al. (1995) from D-array Very Large Arrey (VLA) observations (contours) superimposed on the IRAC $8 \mu \mathrm{m}$ image (grayscale) for a series of correlator channels ranging from a heliocentric velocity of $1285-1120 \mathrm{~km} \mathrm{~s}^{-1}$. The maps show a clear association in the centroid of $\mathrm{HI}$ emission at each velocity with the $8 \mu \mathrm{m}$ PAH-emitting filament. As one proceeds 

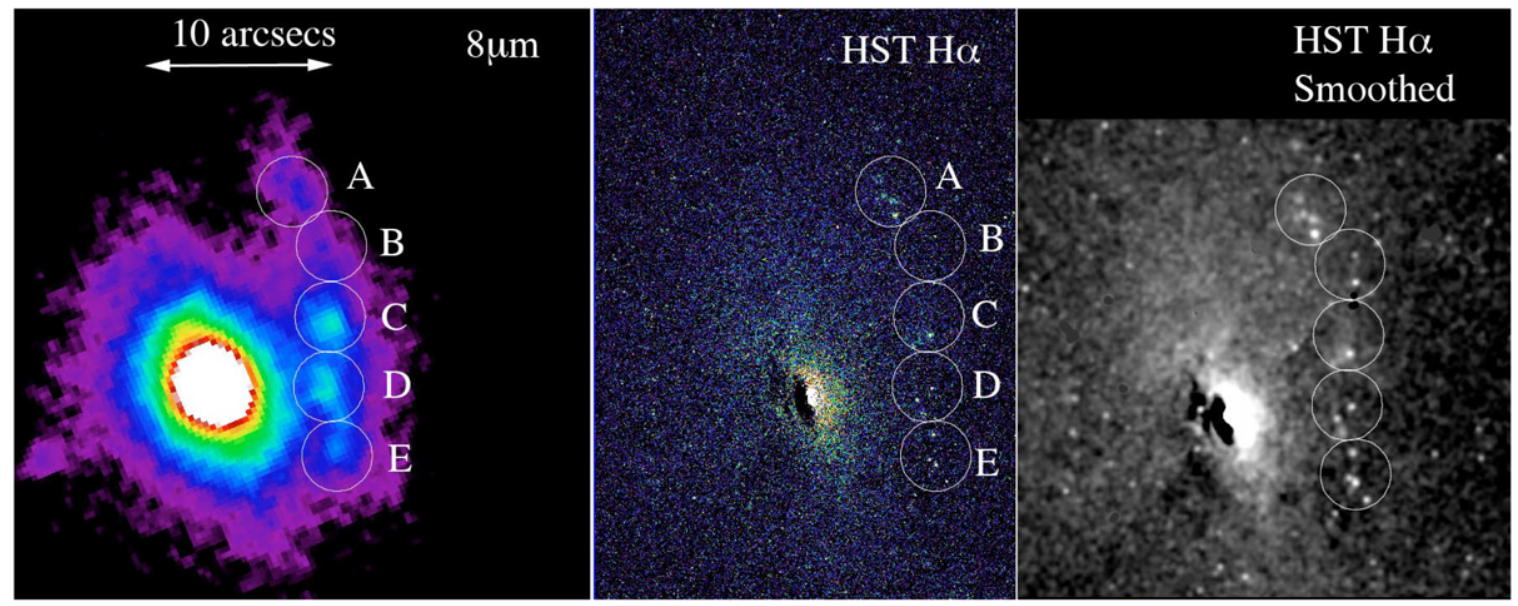

Figure 4. Left: the $8 \mu \mathrm{m}$ IRAC image of NGC 3226 showing areas selected for photometric analysis, A-E (see the text). Middle: H $\alpha$ image of NGC 3226 taken with the HST and showing a series of tiny $\mathrm{H}$ II regions associated with the IRAC blobs. Right: the same $\mathrm{H} \alpha$ image smoothed with a Gaussian to bring out the fainter extended emission. In addition to the $\mathrm{H} \alpha$ regions, significant extended emission is seen, as well as strong dust absorption to the south of the nucleus. (A color version of this figure is available in the online journal.)

Table 2

Observed and Derived IR Properties of Knots in NGC 3226 Filament

\begin{tabular}{|c|c|c|c|c|c|c|}
\hline $\begin{array}{l}\text { Knot } \\
\text { (1) }\end{array}$ & $\begin{array}{c}\mathrm{S}(8 \mu \mathrm{m})_{\text {dust }}{ }^{\mathrm{a}, \mathrm{b}} \\
(\mathrm{mJy}) \\
(2)\end{array}$ & $\begin{array}{c}\mathrm{H} \alpha_{\mathrm{unc}}^{\mathrm{b}, \mathrm{d}} \text { flux } \\
\left(\mathrm{x} 10^{-15} \mathrm{erg} \mathrm{s}^{-1} \mathrm{~cm}^{-2}\right) \\
\text { (3) }\end{array}$ & $\begin{array}{c}\log (\operatorname{L}(8 \mu \mathrm{m}))^{b} \\
\left(\mathrm{erg} \mathrm{s}^{-1}\right) \\
(4)\end{array}$ & $\begin{array}{c}\log \left(L\left(\mathrm{H} \alpha_{\text {unc }}\right)\right)^{\mathrm{b}, \mathrm{d}} \\
\left(\mathrm{erg} \mathrm{s}^{-1}\right) \\
(5)\end{array}$ & $\begin{array}{c}\left.\operatorname{SFR}\left(\mathrm{H} \alpha_{\mathrm{unc}}\right)\right)^{\mathrm{b}, \mathrm{c}} \\
\left(10^{-3} M_{\odot} \mathrm{yr}^{-1}\right) \\
(6)\end{array}$ & $\begin{array}{c}\operatorname{SFR}(8 \mu \mathrm{m}+\mathrm{H} \alpha)^{\mathrm{b}, \mathrm{e}} \\
\left(10^{-3} M_{\odot} \mathrm{yr}^{-1}\right) \\
(7)\end{array}$ \\
\hline A & $0.51(0.1)$ & $1.5(0.15)$ & 39.71 & 38.23 & 0.9 & 1.2 \\
\hline B & $0.42(0.1)$ & $4.7(0.2)$ & 39.63 & 38.73 & 2.8 & 3.1 \\
\hline $\mathrm{C}$ & $0.91(0.1)$ & $5.0(0.2)$ & 39.96 & 38.76 & 3.0 & 3.6 \\
\hline D & $0.82(0.1)$ & $4.6(0.2)$ & 39.92 & 38.72 & 2.8 & 3.3 \\
\hline E & $0.53(0.1)$ & $4.4(0.2)$ & 39.81 & 38.70 & 2.7 & 3.0 \\
\hline
\end{tabular}

Notes.

a $8 \mu \mathrm{m}$ flux density corrected by factor of 1.2 for $\mathrm{r}=3.6$ arcsec aperture correction (Table 5.7 of the IRAC Data Handbook). The flux was also corrected to a "dust+PAH" flux by subtracting $0.25 \times \mathrm{S}(3.6) \mu \mathrm{m}$ flux density to remove the contribution to the band from stellar photospheric emission in the galaxy (see Calzetti et al. 2007).

${ }^{\text {b }}$ Evaluated over aperture radius 3.6 arcsec, or area $=0.24 \mathrm{kpc}^{2}$ at $D=15.1 \mathrm{Mpc}$.

c Assuming SFR $M_{\odot} \mathrm{yr}^{-1}=5.3 \times 10^{-42} \mathrm{~L}(\mathrm{H} \alpha)$ and Kroupa IMF (Kennicutt 1998).

${ }^{\mathrm{d}} \mathrm{H} \alpha$ fluxes (or luminosity) uncorrected for extinction derived from the HST image.

e SFR calculated using the empirical relationship between $\log \left(L\left(\mathrm{H} \alpha_{\text {unc }}\right)+0.011 L(8 \mu \mathrm{m})\right)$ and $\log \left(\mathrm{H} \alpha_{\text {corr }}\right)\left(\right.$ Kennicutt et al. 2009), where $\mathrm{H} \alpha_{\text {unc }}$ is uncorrected for extinction and $\mathrm{H} \alpha$ corr is the unextinguished values, and then converted to SFR using table note (c).

through the channel maps from 1285 to $1120 \mathrm{~km}^{-1}$, we see the centroid following the filament until the H I emission completely disappears at velocities lower than $1120 \mathrm{~km} \mathrm{~s}^{-1}$. At velocities higher than $1285 \mathrm{~km} \mathrm{~s}^{-1}$ (not shown here, but see Mundell et al. 2004), the centroid joins the bulk of the gas that forms the northern H I filament seen in Figure 2. Figure 7 shows two things: (1) that the IRAC $8 \mu \mathrm{m}$ emitting filament is gas rich $\left(M_{H}=9.2 \times 10^{7} M_{\odot}\right.$ for $D=15.1 \mathrm{Mpc}$ and integrating over the range of H I channels shown in Figure 7), with the center of the filament having a systemic velocity of $1202 \mathrm{~km} \mathrm{~s}^{-1}$, and (2) the star formation seen in this dusty structure seems to represents the southern tip and kinematic termination of the large northern $\mathrm{HI}$ plume. The termination of the northern $\mathrm{HI}$ plume deep inside the inner regions of NGC 3226 may suggest "late-stage" accretion onto the galaxy.

\subsection{Mid-IR Spectral Properties of NGC 3226}

In Figure 8, we show the IRS-SL spectrum of NGC 3226 extracted from the spectral cube. The galaxy shows PAH emission and emission from the $0-0 \mathrm{~S}(3) \lambda 9.66 \mu \mathrm{m}$ rotational line of warm molecular hydrogen. In Table 3, we present the band and line fluxes for the well-determined PAH features and the $0-0 \mathrm{~S}(3)$ line determined from the application of PAHFIT (Smith et al. 2007b). The large value of the 11.3/7.7 (=0.67) $\mathrm{PAH}$-feature power ratio and small value of the 6.2/7.7 PAH $(=0.22)$ ratio places NGC 3226 in the region of neutral PAHs with long carbon chains $(N \sim 300$; Draine \& Li 2001). This may relate to a relatively quiescent UV environment in NGC 3226 due to its low SFR and weak AGN properties.

In Figure 9, we show the high-resolution spectrum of NGC 3226 obtained with the IRS. Unlike the SL spectrum, a dedicated background observation off the target was not made for this archival observation (taken early in the mission). Therefore, there is an unknown offset in the spectrum from zodiacal light contamination. However, the line fluxes themselves are not affected. The SH spectrum, extending to $19.5 \mu \mathrm{m}$, was scaled upward (in the figure by a factor of four) so that its continuum matched the overlapping continuum of the LH spectrum, which has a larger slit. Line fluxes are provided in Table 3. Figure 9 shows strong emission from the rotational $\mathrm{H}_{2}$ transition $0-0 \mathrm{~S}(1) 17.03 \mu \mathrm{m}$ and well as weaker emission from $0-0 \mathrm{~S}(2) 12.28$ and $\mathrm{S}(0) 28.22 \mu \mathrm{m}$. Atomic fine-structure lines of 

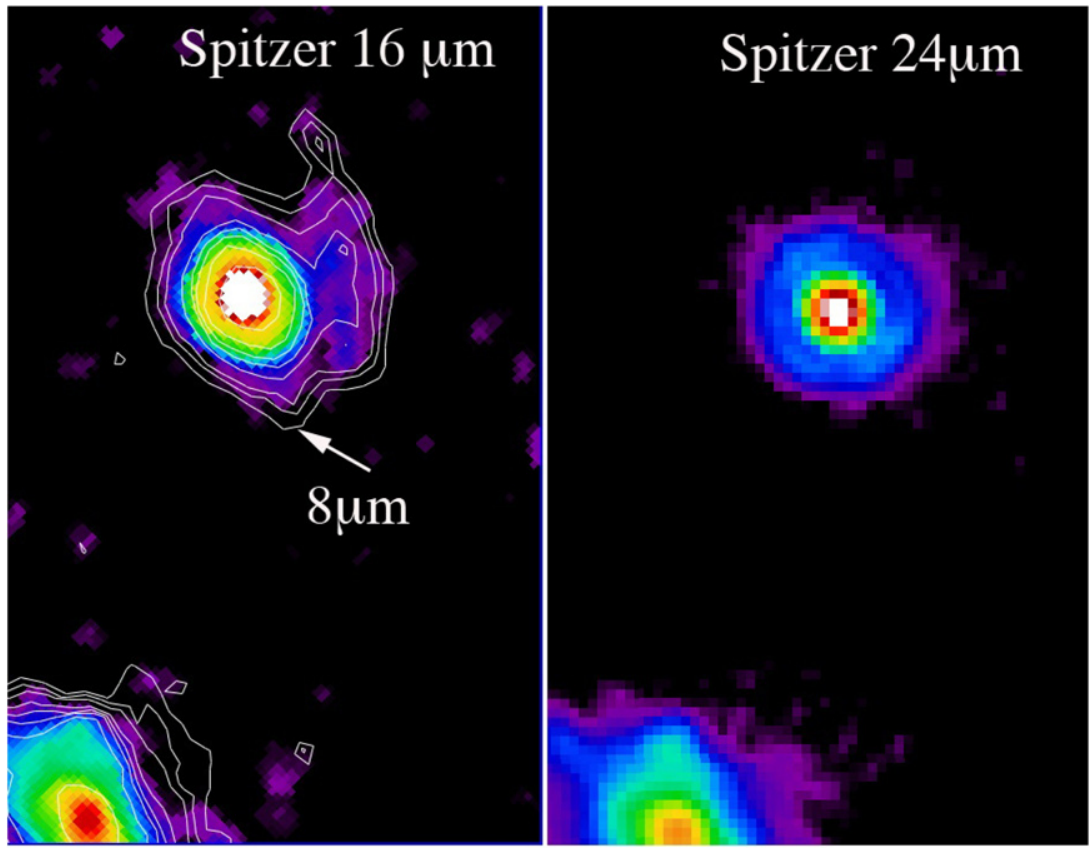

Figure 5. Left: contours of $8 \mu \mathrm{m}$ IRAC emission superimposed on a $16 \mu \mathrm{m}$ IRS peak-up image of the galaxy. Note the close association between the $8 \mu \mathrm{m}$ contours and the plume. Right: the $24 \mu \mathrm{m}$ image of the same region. Note the absence of dust emission from the plume. This suggests that the $16 \mu \mathrm{m}$ emission from the plume contains either strong 0-0S (1) $\mathrm{H}_{2}$ emission at $17 \mu \mathrm{m}$ or $16 \mu \mathrm{m}$ PAH emission, or both. IRS spectra, which do not cover this region, show unusually strong $\mathrm{H}_{2}$ emission from the center of the galaxy.

(A color version of this figure is available in the online journal.)

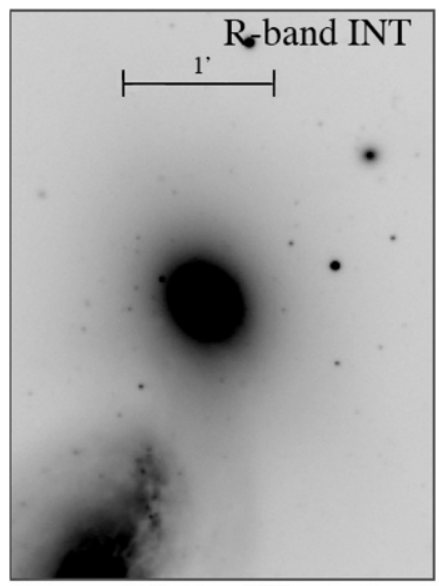

$250 \mu \mathrm{m}$ Herschel

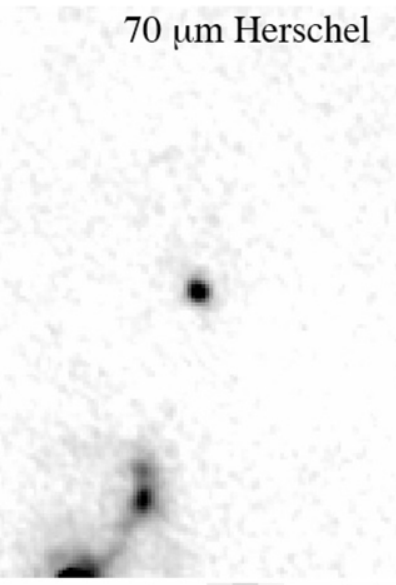

$350 \mu \mathrm{m}$ Herschel

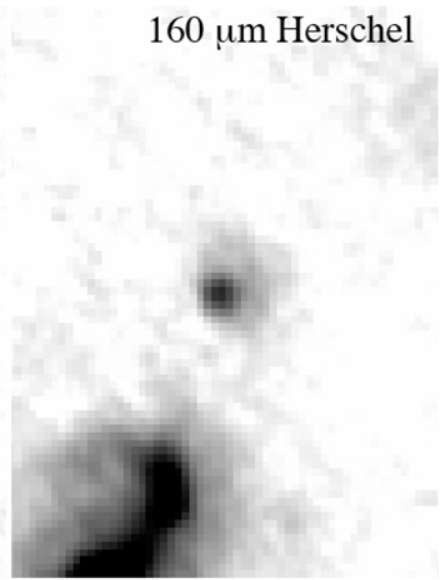

$500 \mu \mathrm{m}$ Herschel
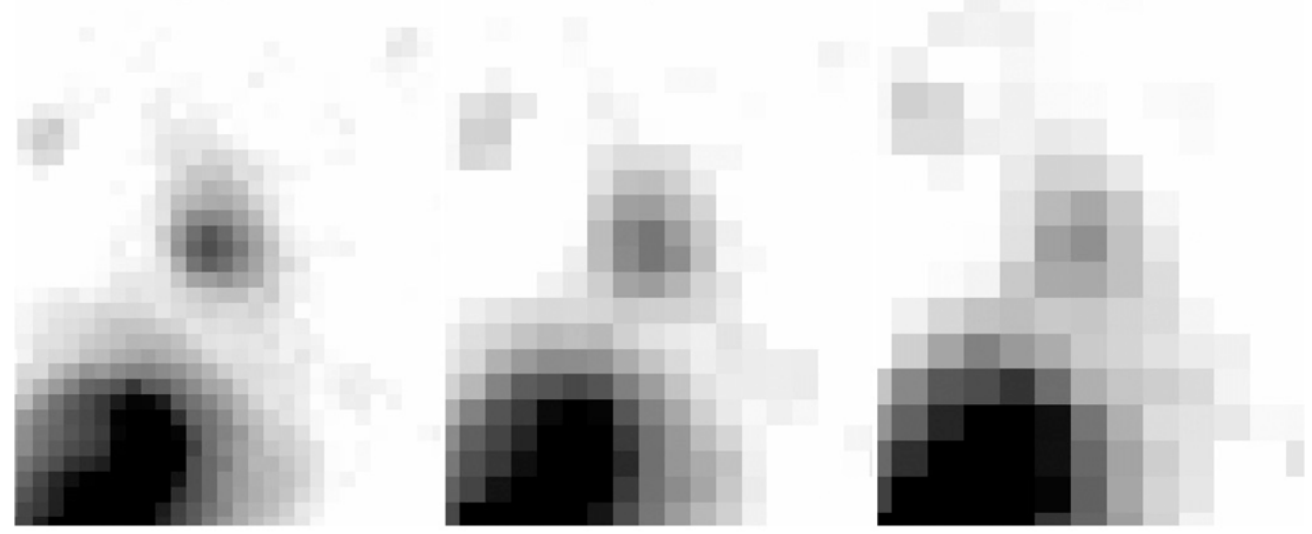

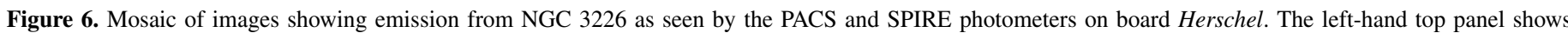

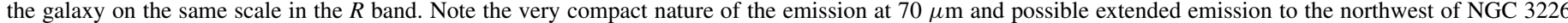

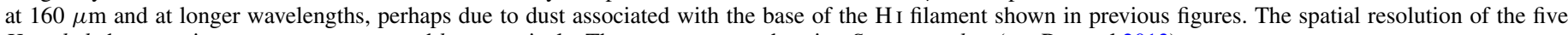
Herschel photometric maps are $x, y, z, a$, and $b$, respectively. The maps were made using Scanamorphos (see Roussel 2013). 


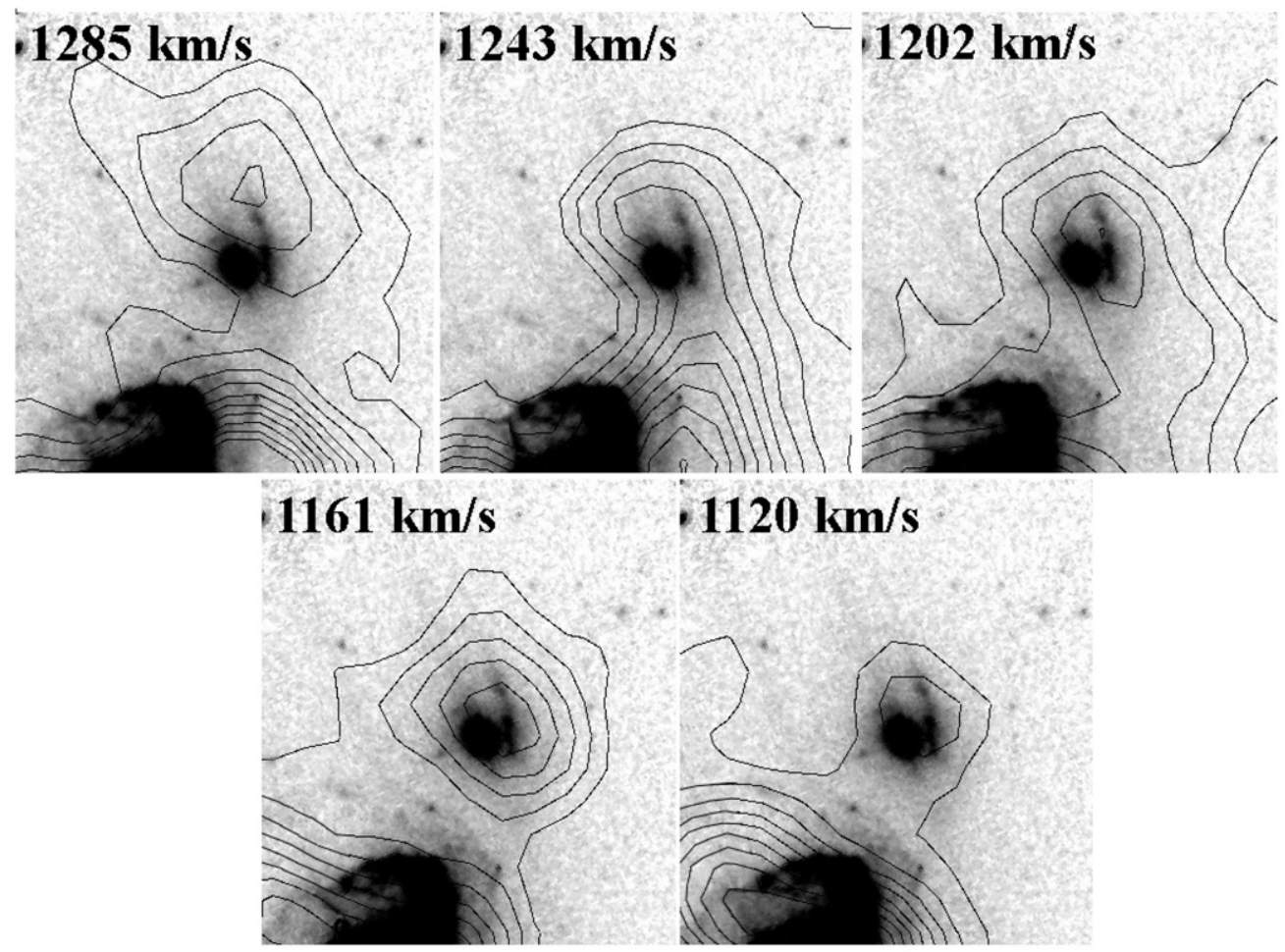

Figure 7. Neutral hydrogen emission from $40 \mathrm{~km} \mathrm{~s}^{-1}$ wide channels (contours) superimposed on the IRAC $8 \mu \mathrm{m}$ emission (grayscale) of NGC 3226 . The figure shows data obtained from Mundell et al. (1995) D-array VLA data. The sequence starts at $1285 \mathrm{~km} \mathrm{~s}^{-1}$ (heliocentric velocity) and shows that as the velocity decreases, there is a clear association of the H I centroid with the $8 \mu \mathrm{m}$ emitting PAH emission. Note how, at the lowest velocity, the H I terminates in the $8 \mu \mathrm{m}$ plume and does not extend beyond the base of that plume, suggesting that the northern $\mathrm{H}$ i plume actually ends at the $8 \mu \mathrm{m}$ feature. Channel maps showing $\mathrm{H}$ i at even higher velocities than shown here continue up into the much more extended northern H I plume of NGC 3226 (Mundell et al. 1995).

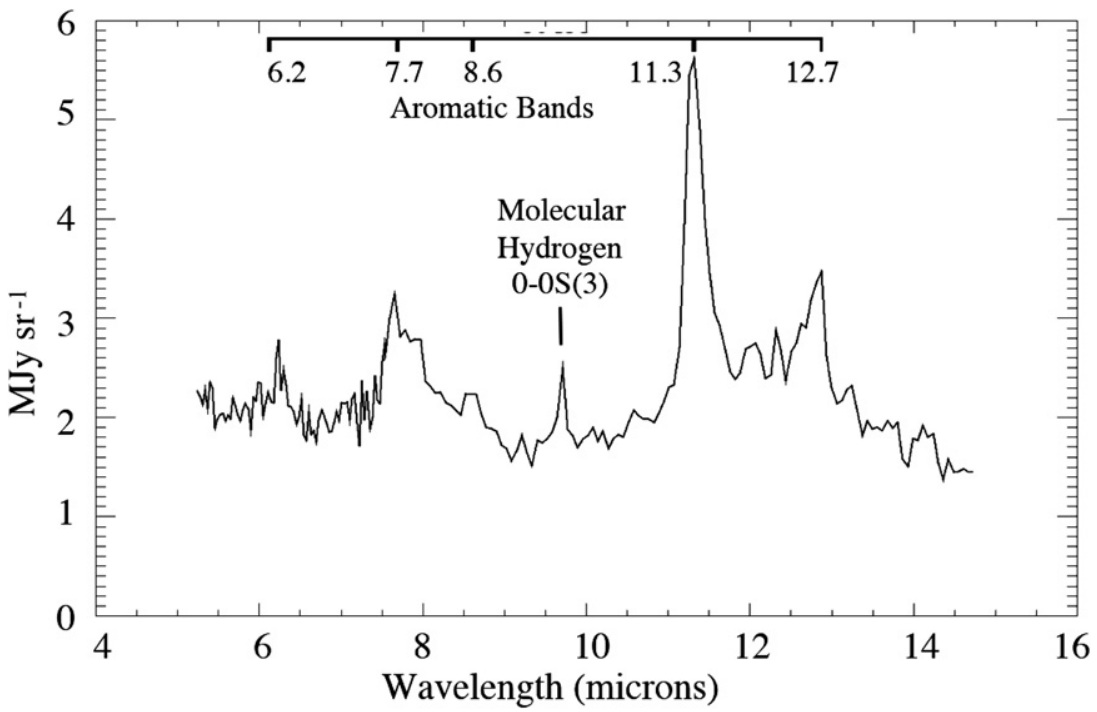

Figure 8. Spectrum of NGC 3226 made with the short-low (SL) module of the Spitzer IRS extracted from a $22 \times 16 \operatorname{arcsec}^{2}$ area in the center of a small map of NGC 3226. In addition to the broad PAH features at 6.2, 7.7, 8.6, 11.3, and $12.7 \mu \mathrm{m}$, molecular hydrogen is detected in the $0-0 \mathrm{~S}(3) 9.66 \mu \mathrm{m}$ line.

$[\mathrm{Ne}$ II] $12.8 \mu \mathrm{m}$, [Ne III] $15.6 \mu \mathrm{m}$, and [Si II] $34.81 \mu \mathrm{m}$ as well as PAH emission are also seen in the spectrum. These observations were taken against a relatively high zodiacal light background (NGC 3226 is only nine degrees from the ecliptic plane), and therefore the absolute scaling (and possibly the shape) of the continuum is likely affected by extra (zodiacal) background emission. The measurement of the strength of the emission lines is not affected by zodiacal light.

\section{WARM $\mathrm{H}_{2}$ PROPERTIES}

The discovery of strong pure-rotational emission lines of molecular hydrogen in NGC 3226, implies the existence of warm $(T>100 \mathrm{~K}) \mathrm{H}_{2}$ in the galaxy. Temperatures significantly below $100 \mathrm{~K}$ are not capable of exciting even the lowest level transition which gives rise to the $\lambda 28 \mu \mathrm{m}$ line. If several emission lines are detected, it is possible to gain some idea of the temperature and total column density of gas by creating a $\mathrm{H}_{2}$ 


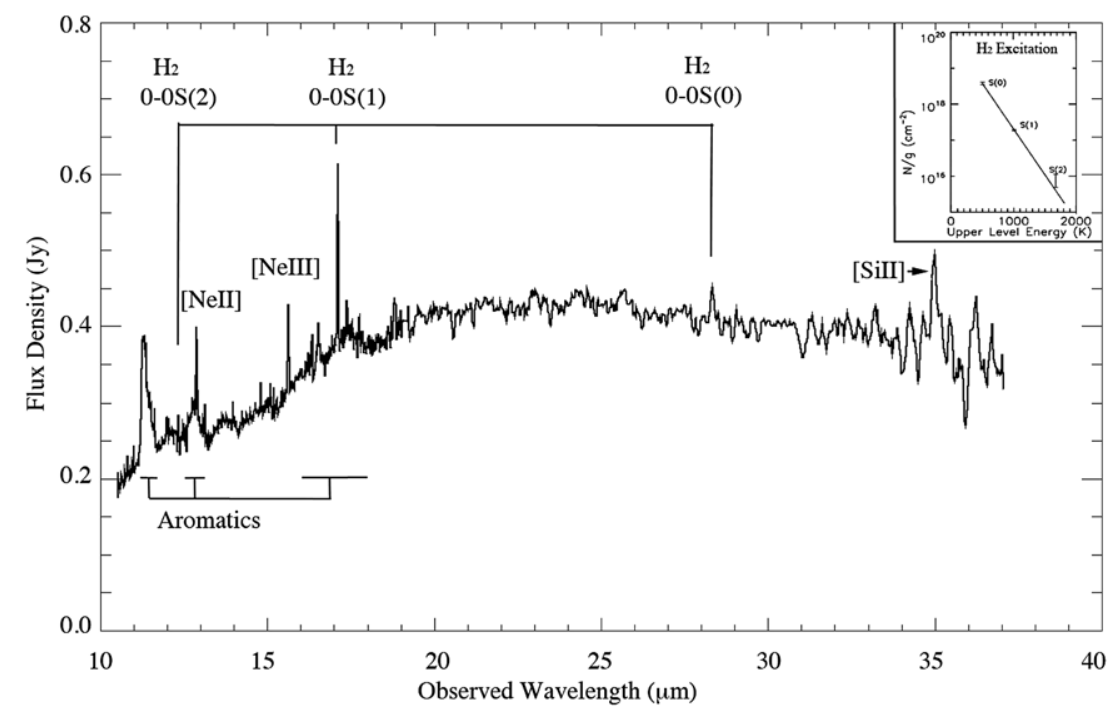

Figure 9. High-resolution (SH and LH) IRS spectrum of the center of NGC 3226 showing clear evidence of strong molecular hydrogen, PAH emission, and other emission lines. Note the flattening of the continuum beyond $20 \mu \mathrm{m}$, typical of low-luminosity AGNs (Ogle et al. 2010). The inset shows the excitation diagram for molecular hydrogen and a single temperature fit to the points under the assumption of fully extended $\mathrm{H}_{2}$ emission on the scale of the LH IRS slit, indicating a temperature for the $\mathrm{H}_{2}$ of $T=144 \mathrm{~K}$. However, a more realistic assumption is that it is of intermediate scale (10-15 arcsec) and the temperature under these assumptions is found to be lower $(T=124 \mathrm{~K}$, see the text). Note that there is an unknown contribution to the continuum by zodiacal light that was not removed from the spectrum.

Table 3

Extracted Line and PAH Band Fluxes from Spitzer IRS (SL, SH, and LH) in units $10^{-17} \mathrm{~W} \mathrm{~m}^{-2}$

\begin{tabular}{|c|c|c|c|c|c|c|c|}
\hline IRS Module & $6.2 \mu \mathrm{m}$ PAH & $7.7 \mu \mathrm{m}$ РAH & $11.3 \mu \mathrm{m}$ PAH & $12.6 \mu \mathrm{m}$ PAH & $0-0 \mathrm{~S}(3) 9.7 \mu \mathrm{m}$ & $11.3 / 7.7$ & $6.2 / 7.7$ \\
\hline $\mathrm{SL}^{\mathrm{a}}$ & $15.0(1.5)$ & $67.1(6.7)$ & $44.6(4.5)$ & $19.9(2.0)$ & $2.35(0.3)$ & 0.66 & 0.22 \\
\hline IRS Module & $0-0 \mathrm{~S}(0) 28.2 \mu \mathrm{m}$ & $0-0 \mathrm{~S}(1) 17.0 \mu \mathrm{m}$ & $0-0 \mathrm{~S}(2) 12.3 \mu \mathrm{m}$ & $11.3 \mu \mathrm{m} \mathrm{PAH}$ & {$[\mathrm{Ne} \mathrm{II}] 12.8 \mu \mathrm{m}$} & {$[\mathrm{Ne}$ III] $15.5 \mu \mathrm{m}$} & {$[\mathrm{Si} \mathrm{II}] 34.8 \mu \mathrm{m}$} \\
\hline LH & $1.8(0.15)$ & $\ldots$ & $\ldots$ & $\ldots$ & $\ldots$ & $\ldots$ & $2.53(0.29)$ \\
\hline $\mathrm{SH}^{\mathrm{c}}$ (Preferred) & $\ldots$ & $4.6(0.2)$ & $0.75(0.3)$ & $47(6)$ & $4.0(0.34)$ & $2.8(0.12)$ & $\ldots$ \\
\hline
\end{tabular}

Notes.

${ }^{a}$ Integrated over an area of $22 \times 16$ arcsec centered on the $\mathrm{H}_{2}$ disk and capturing most of its emission. Line fluxes were measured using PAHFIT (Smith et al. 2007b).

${ }^{b}$ Assumes the $\mathrm{H}_{2}$ is extended on the scale of the LH slit $\left(22 \times 11 \operatorname{arcsec}^{2}\right)$ and SH scaled by 4.1 to LH.

${ }^{c}$ Assumes the $\mathrm{H}_{2}$ is extended on a more realistic intermediate scale (approximately $15 \times 15 \operatorname{arcsec}^{2}$ ), and SH scaled by 2.0 to LH. Supported by similarity in PAH $11.3 \mu \mathrm{m}$ flux as measured in SL and SH, as well as the measured scale of the $\mathrm{H}_{2}$ seen in Figure 10.

excitation diagram: a plot of the upper-level transition column density normalized by its statistical weight $N_{u} / \mathrm{g}_{u}$, against the upper-level transition energy $\mathrm{E}_{u}$. If the gas is in thermal equilibrium, the ortho- and para-branches of the $\mathrm{H}_{2}$ energy level diagram contribute points in the excitation diagram that fall on a straight line, the slope of which is inversely proportional to the temperature (for more details, see Rigopoulou et al. 2002; Roussel et al. 2007).

The inset to Figure 9 shows the excitation diagram derived from the $\mathrm{SH} / \mathrm{LH}$ spectra and are based on the line fluxes (Table 3) for the three lowest energy rotational transitions $(0-0 \mathrm{~S}(0), \mathrm{S}(1)$, and $\mathrm{S}(2))$. We also provide line fluxes for other detected atomic lines in Table 3 . We estimate a range of temperatures and total $\mathrm{H}_{2}$ masses of the warm molecular hydrogen. Assuming an ortho-to-para $\mathrm{H}_{2}$ ratio appropriate for LTE, we derive a temperature range of 144-173 $\mathrm{K}$ and a warm $\mathrm{H}_{2}$ mass of $1.1-0.7 \times 10^{7} M_{\odot}$ in the LH aperture $\left(1.6 \times 0.8 \mathrm{kpc}^{2}\right)$. The range of temperatures and masses takes into account both observational uncertainty as well as uncertainty in the relative scaling of the SH and LH spectra, which depends on the assumed $\mathrm{H}_{2}$ distribution.

Deep single-dish IRAM $30 \mathrm{~m}$ CO observations by Young et al. (2011) failed to detect molecular hydrogen down to a level of $<1.7 \times 10^{7} M_{\odot}$ for a nominal velocity width of $300 \mathrm{~km} \mathrm{~s}^{-1}$ and a $3 \sigma$ upper limit and assuming $X_{\mathrm{CO}}=N\left(\mathrm{H}_{2}\right) / I(\mathrm{CO})_{1-0}$ $=2 \times 10^{20} \mathrm{~cm}^{-2}\left(\mathrm{~K} \mathrm{~km} \mathrm{~s}^{-1}\right)^{-1}$. Assuming the $\mathrm{CO}$ is not very extended on the scale of the 22 arcsec IRAM beam and using this $\mathrm{X}_{\mathrm{CO}}$ factor would imply a warm-to-total $\mathrm{H}_{2}$ mass ratio of $M\left(\mathrm{H}_{2}\right)_{w} / M\left(\mathrm{H}_{2}\right)_{\text {total }}>0.29-0.39$.

Roussel et al. (2007) investigated the $M\left(H_{2}\right)_{w} / M\left(\mathrm{H}_{2}\right)_{\text {total }}$ ratio for the SINGs sample and found that it decreased with $\mathrm{H}_{2}$ excitation temperature-ranging from 0.5 to 0.02 , with a median value of $\sim 0.15$ (correcting for a slightly different assumed $\mathrm{X}_{\mathrm{CO}}$ ratio). It was also found that the temperature of the warm $\mathrm{H}_{2}$ in LINERs and Seyferts was systematically higher than in $\mathrm{H}$ II region-dominated galaxies and the $M\left(\mathrm{H}_{2}\right)_{w} / M\left(\mathrm{H}_{2}\right)_{\text {total }}$ ratio was generally lower in these galaxies compared with $\mathrm{H}$ IIdominated galaxies.

NGC 3226 lies on the upper end of the distribution of $M\left(\mathrm{H}_{2}\right)_{w} / M\left(\mathrm{H}_{2}\right)_{\text {total }}$ ratios for H II-dominated galaxies but is an outlier for LINERs and Seyferts, having more warm $\mathrm{H}_{2}$ than expected for its temperature. The intergalactic filament in Stephan's Quintet (Guillard et al. 2012) has also been shown to exhibit a high fraction of warm $\mathrm{H}_{2}$, and in that case, there is strong evidence that the filament is strongly heated by mechanical energy from shocks (see also Appleton et al. 2013). 


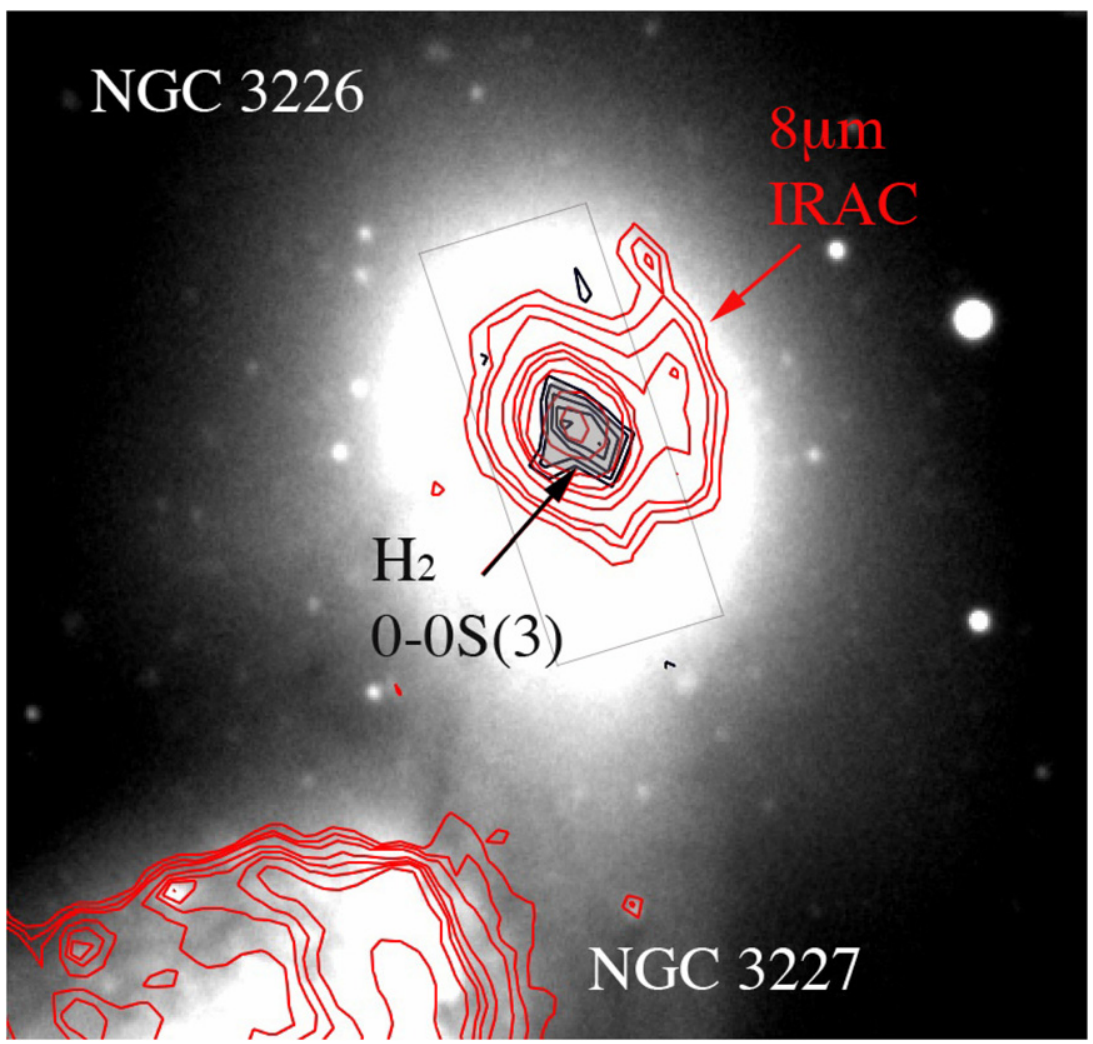

Figure 10. Contours of $8 \mu \mathrm{m}$ IRAC emission (red), and 0-0 S (3)9.66 $\mu \mathrm{m}$ molecular hydrogen (black/gray filled) superimposed on a $B$-band visible light CCD image of NGC 3226 (and NGC 3227 to the south). The gray box shows the area covered in the spectra mapping of NGC 3226 by the SL Spitzer IRS instrument. Note that the area mapped did not include the plume feature. Details of the mapping are given in the main text.

(A color version of this figure is available in the online journal.)

We caution, however, against overinterpreting the significance of the warm molecular fraction. A comparison of this ratio between galaxies is only meaningful if the value of $\mathrm{X}_{\mathrm{CO}}$ is the same in NGC 3226 as that in other galaxies, including the Milky Way (see Bolatto et al. 2013, for a complete discussion).

To explore the distribution of the $\mathrm{H}_{2}$ in NGC 3226 , we created a spectral cube from spectral maps made with the IRS SL data using the CUBISM software of Smith et al. (2007b). From that cube we extracted a map of the $0-0 \mathrm{~S}(3) 9.66 \mu \mathrm{m} \mathrm{H}_{2}$ line, after removing a local continuum. This is shown (black contours) in Figure 10 along with the IRAC $8 \mu \mathrm{m}$ contours (red contours), both superimposed against a $B$-band image of the galaxy. The emission from $\mathrm{H}_{2}$ is confined to an elongated structure with a P.A. $\sim 45^{\circ}$ and on a smaller scale than the PAH emission (which is much more extensive and symmetrically distributed).

It is reasonable to ask what the source of heating of the warm $\mathrm{H}_{2}$ emission might be. One possibility is that the $\mathrm{H}_{2}$ is heated by the AGN known to be at the center of NGC 3226. The X-ray luminosity of the nuclear source (George et al. 2001; Ho 2009) is 4-7 $\times 10^{39} \mathrm{erg} \mathrm{s}^{-1}$ (scaled to $D=15.1 \mathrm{Mpc}$ ). Summing just the warm $\mathrm{H}_{2}$ line for the $0-0 \mathrm{~S}(0), \mathrm{S}(1)$, and $\mathrm{S}(2)$ lines gives a warm $\mathrm{H}_{2}$ line luminosity in the range of $2.010^{39} \mathrm{erg} \mathrm{s}^{-1}<$ $\mathrm{L}\left(\mathrm{H}_{2}\right)<$ is $3.410^{39} \mathrm{erg} \mathrm{s}^{-1}$, which would imply an impossibly high X-ray gas heating efficiency (the efficiency is expected to be significantly less than 10\% (Lepp \& McCray 1983)). We can therefore rule out X-ray heating of the $\mathrm{H}_{2}$.

A common explanation for the detection of emission from the rotational lines of $\mathrm{H}_{2}$ in normal galaxies is through photoelectric heating from small grains and PAH molecules excited in photo-dissociation regions (PDRs) associated with young stars. However, for NGC 3226, we estimate the $\mathrm{H}_{2} / \mathrm{PAH}(7.7 \mu \mathrm{m}$ ratio $)$ to be 0.06 , which places it in the class of galaxies that exceeds the efficiency of $\mathrm{H}_{2}$ heating by PDRs (see Roussel et al. 2007) by a large factor (the median value for this ratio for SINGS galaxies is 0.0086). Guillard et al. (2012) used the Meudon PDR code $^{14}$ to demonstrate that over a wide range of UV excitation and densities in PDRs, the $L\left(\mathrm{H}_{2} \mathrm{~S}(0)-\mathrm{S}(3)\right) / L(\mathrm{PAH} 7.7)$ ratio cannot exceed 0.04. Ratios exceeding this value must have an additional heating source, such as the dissipation of mechanical energy through shocks and turbulence or very high cosmic-ray energy densities.

Evidence that shocks can create very strong heating of $\mathrm{H}_{2}$ and can boost the far-IR cooling from the [C II] $158 \mu \mathrm{m}$ line has been presented for the intergalactic filament in the Stephan's Quintet compact group (Appleton et al. 2006; Guillard et al. 2009; Cluver et al. 2010; Appleton et al. 2013), the bridge between the "Taffy" galaxies (Peterson et al. 2012), and in other Hickson compact groups (HCGs; Cluver et al. 2013). Ogle et al. (2010) defined galaxies with $\mathrm{H}_{2} / \mathrm{PAH}$ ratios $>0.04$ as molecular hydrogen emission line galaxies (MOHEGS). Like other MOHEGs, the warm $\mathrm{H}_{2}$ in NGC 3226 is likely heated by mechanical energy through shocks and turbulence. PDR heating must play a minor role, especially given the very low star-formation rates we find for this galaxy.

One possibility is that the gas is shock-heated through energy deposited by infall from the $\mathrm{HI}$ plume. For a mass infall rate from the plume of $d m / d t=1 M_{\odot} \mathrm{yr}^{-1}$ falling from a distance of $r \approx 10 \mathrm{kpc}$ and assuming a mass within that radius of $M(r)=10^{11} M_{\odot}$, the energy input rate would be $d E / d t=(G M(r) / r) d m / d t \sim 3 \times$

\footnotetext{
14 See http://pdr.obspm.fr/PDRcode.html for details.
} 

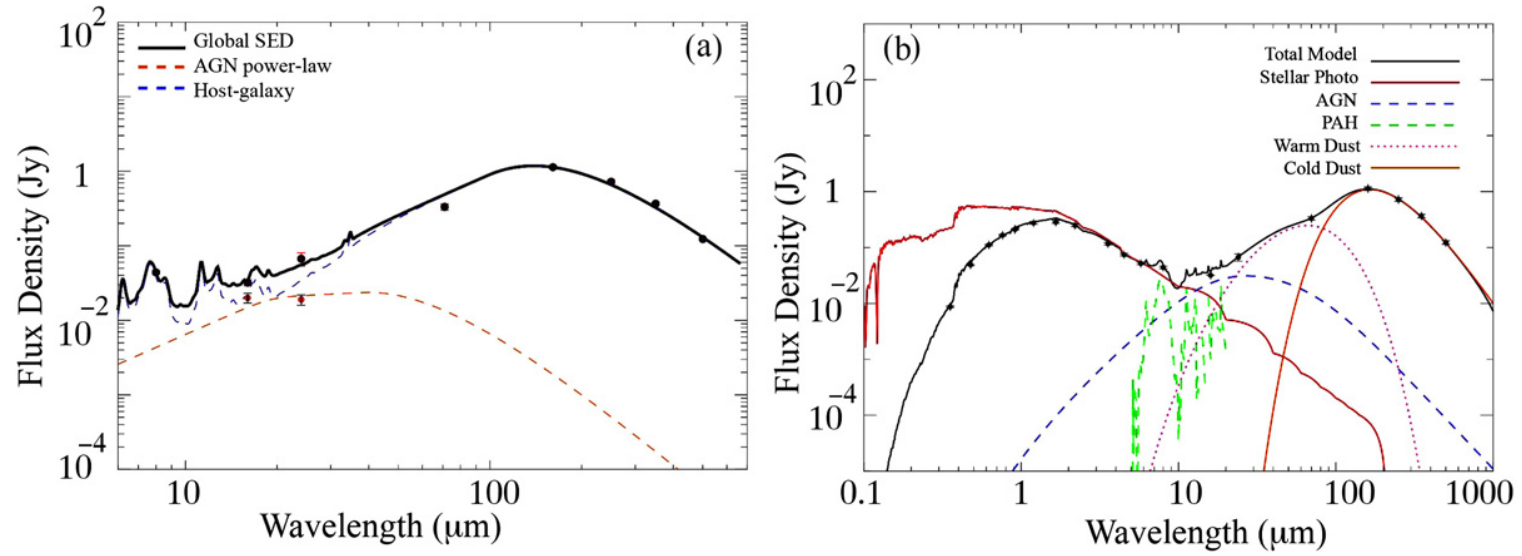

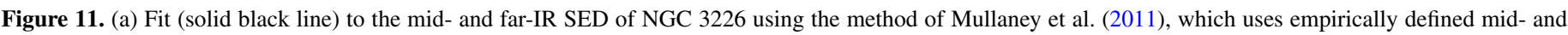

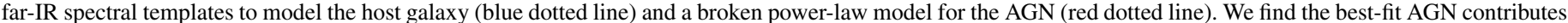

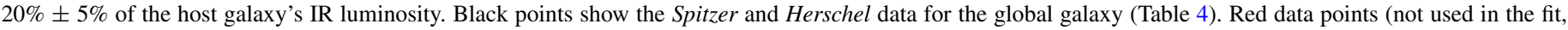

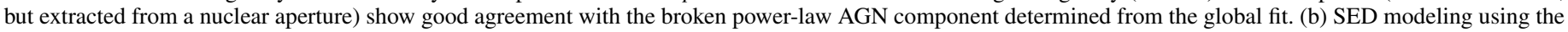

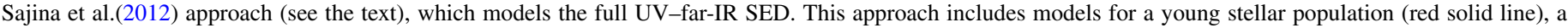

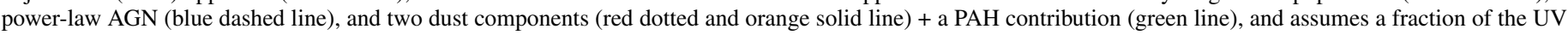

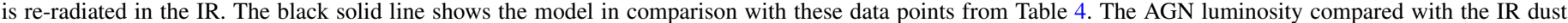
luminosity is similar to the previous modeling ( $18 \% \pm 4 \%$ ), but additionally allows several key parameters of the host galaxy to be quantified (see Table 5).

(A color version of this figure is available in the online journal.)

$10^{33} \mathrm{~W}\left(3 \times 10^{40} \mathrm{erg} \mathrm{s}^{-1}\right)$, which is enough energy to heat the warm $\mathrm{H}_{2}$ with a $10 \%$ efficiency.

We cannot completely rule out a role in the shock-heating of the $\mathrm{H}_{2}$ by the AGN. Recently, Ogle et al. (2014) have shown that even the weak radio jet in NGC 4258 is capable of shock-heating large quantities of molecular hydrogen in that galaxy. NGC 3226 contains a $3.9 \mathrm{mJy}$ flat or inverted spectrum radio source at $5 \mathrm{GHz}$ (Filho et al. 2006), which is compact on milli-arcsecond scales (Nagar Falcke \& Wilson 2005). However, unlike NGC 4258, there is no direct indication that any radio jet in the system is directly interacting with the host galaxy (in NGC 4258, the so-called anomalous arms are very obvious at many wavelengths).

\section{THE SPECTRAL ENERGY DISTRIBUTION OF NGC 3226}

\subsection{AGN Contribution to IR}

NGC 3226 is known to contain a low-luminosity AGN, and this might manifest itself in the spectral energy distribution (SED) of the galaxy shown in Figures 11(a) and (b) (the flux densities plotted are presented in Table 4). We first consider the infrared component of the SED derived from Spitzer (IRAC and MIPS $24 \mu \mathrm{m})$ and Herschel far-IR photometry (70, 120, 250,350 , and $500 \mu \mathrm{m}$ ) derived from a 54 arcsecond diameter aperture covering the whole galaxy. Figure 11(a) shows the observed SED and a model decomposition of the SED into an AGN and host galaxy component using the method of Mullaney et al. (2011). The modeling software developed by these authors, "DECOMPIR," uses empirical templates for the host galaxy SED, plus an intrinsic AGN broken power-law model to fit the SED using a minimization of the least-squares algorithm. In Figure 11(a), we show the best result from the fitting, in which the AGN fraction of the IR emission, $L(\mathrm{AGN}) / L$ (FIR), is $20 \% \pm 5 \%$. Because of the limited number of empirical host galaxy templates used in this modeling, the final composite spectrum slightly overestimates the $70 \mu \mathrm{m}$ contribution for the galaxy.
In order to try to refine the fit, we perform additional modeling using the method of Sajina et al. $(2006,2012)$ that takes into account the full UV-IR SED shown in Figure 11(b). The additional points are from GALEX (FUV and NUV) sky survey images, SDSS images $(u, g, r, i), 2 \mathrm{MASS}\left(j, h\right.$ and $\left.k_{s}\right)$, and the Spitzer and Herschel data already discussed. As with the previous modeling, a power-law form is assumed for the AGN component. However, instead of a template for the host galaxy, the galaxy is built up from various model components. These include a stellar photospheric component that assumes a 100 Myr single population model from Maraston (2005) and a warm and cold dust component from Sajina et al. (2006), together with the modified PAH template of (Lacy et al. 2007). Figure 11(b) presents the least-squares fit of the model to the observed fluxes (black solid line). The red line shows the unattenuated stellar spectrum found to be most consistent with the UV through IR data. Parameters resulting from the model fit are presented in Table 5, including the estimated extinction, star formation rate, dust mass, and stellar mass consistent with the SED. The derived AGN fraction of the far-IR emission is found to be $18 \pm 4 \%$, very similar to that found using Mullaney et al. (2011) model.

We emphasize that although we have fitted the mid-IR emission with an assumed AGN component, we cannot conclusively prove that the mid-IR component is caused by the AGN. Evidence that the AGN model has some validity comes when we overplot on Figure 11(a) (we do not fit) the $16 \mu \mathrm{m}$ and $24 \mu \mathrm{m}$ photometry obtained in a small nuclear aperture (diameter 10 arcsec) on the same plot (red points). They agree very closely with the AGN model, suggesting that the mid-IR component is strongly peaked - as would be the case for an AGN. Previous modeling of NGC 3226 using an earlier version of the SED (without the GALEX or the $16 \mu \mathrm{m}$ peak-up data) that did not include an AGN has been performed by Lanz et al. (2013). These authors used a MAGPHYS model (da Cunha et al. 2008) that assumes all the IR components result from various dusty ISM components. However, as with our modeling, Lanz et al. (2013) found that a hot dust component was needed to explain mid-IR component of the SED, but this model attributes the 
Table 4

Observed Flux Density of NGC 3226 from UV to Far-IR and $1 \sigma$ Uncertainties - 54 arcsec Aperture

\begin{tabular}{|c|c|c|c|c|c|c|c|}
\hline $\begin{array}{l}\mathrm{UVW}^{\mathrm{a}} \\
(\mathrm{mJy})\end{array}$ & $\begin{array}{l}\mathrm{NUV}^{\mathrm{b}} \\
(\mathrm{mJy})\end{array}$ & $\begin{array}{l}\mathrm{UVW}^{\mathrm{a}} \\
(\mathrm{mJy})\end{array}$ & $\begin{array}{c}\mathrm{SDSS}_{u}{ }^{\mathrm{c}} \\
(\mathrm{mJy})\end{array}$ & $\begin{array}{c}\mathrm{SDSS}_{g}{ }^{\mathrm{c}} \\
(\mathrm{mJy})\end{array}$ & $\begin{array}{c}\mathrm{SDSS}_{r}{ }^{\mathrm{c}} \\
(\mathrm{mJy})\end{array}$ & $\begin{array}{c}\mathrm{SDSS}_{i}{ }^{\mathrm{c}} \\
(\mathrm{mJy})\end{array}$ & $\begin{array}{c}\mathrm{SDSS}_{z}{ }^{\mathrm{c}} \\
(\mathrm{mJy})\end{array}$ \\
\hline $0.86(0.09)$ & $1.24(0.12)$ & $2.65(0.39)$ & $8.71(0.45)$ & $49.2(2.0)$ & $110.7(6.0)$ & $166(9)$ & $211(10)$ \\
\hline $\begin{array}{l}\mathrm{J}^{\mathrm{d}} \\
(\mathrm{mJy})\end{array}$ & $\begin{array}{c}\mathrm{H}^{d} \\
(\mathrm{mJy})\end{array}$ & $\begin{array}{c}\mathrm{K}_{s}^{\mathrm{d}} \\
(\mathrm{mJy})\end{array}$ & $\begin{array}{c}\text { IRAC-3.6 } \mu \mathrm{m}^{\mathrm{e}} \\
(\mathrm{mJy})\end{array}$ & $\begin{array}{c}\text { IRAC-4.5 } \mu \mathrm{m}^{\mathrm{e}} \\
(\mathrm{mJy})\end{array}$ & $\begin{array}{c}\text { IRAC-5.8 } \mu \mathrm{m}^{\mathrm{e}} \\
(\mathrm{mJy})\end{array}$ & $\begin{array}{c}\text { IRAC-8 } \mu \mathrm{m}^{\mathrm{e}} \\
(\mathrm{mJy})\end{array}$ & $\begin{array}{c}\text { IRS-16 } \mu \mathrm{m}^{\mathrm{e}} \\
(\mathrm{mJy})\end{array}$ \\
\hline $275(25)$ & $286(28)$ & $249(25)$ & $117(7)$ & $74(4)$ & $51.9(2.8)$ & $44.0(1.4)$ & $32(6)$ \\
\hline $\begin{array}{l}\text { MIPS-24 } \mu \mathrm{m}^{\mathrm{e}} \\
(\mathrm{mJy})\end{array}$ & $\begin{array}{c}\text { PACS-70 } \mu \mathrm{m}^{\mathrm{e}} \\
(\mathrm{mJy})\end{array}$ & $\begin{array}{c}\text { PACS-160 } \mu \mathrm{m}^{\mathrm{e}} \\
(\mathrm{mJy})\end{array}$ & $\begin{array}{c}\text { SPIRE-250 } \mu \mathrm{m}^{\mathrm{e}} \\
(\mathrm{mJy})\end{array}$ & $\begin{array}{c}\text { SPIRE-350 } \mu \mathrm{m}^{\mathrm{e}} \\
(\mathrm{mJy})\end{array}$ & $\begin{array}{c}\text { SPIRE-500 } \mu \mathrm{m}^{\mathrm{e}} \\
(\mathrm{mJy})\end{array}$ & & \\
\hline $67(7)$ & $332(17)$ & $1133(56)$ & $722(4)$ & $367(15)$ & $123(10)$ & & \\
\hline
\end{tabular}

Notes.

${ }^{\text {a }}$ From the $S W I F T$ satellite survey.

b Derived from image from the GALEX sky survey.

${ }^{c}$ Data obtained from the SLOAN Digital Sky Survey.

${ }^{\mathrm{d}}$ Data from 2MASS.

e Data from this paper.

Table 5

Derived Properties of NGC 3226 from SED Fitting

\begin{tabular}{|c|c|c|c|c|c|c|c|c|c|}
\hline Source & $\tau_{v}$ & $\begin{array}{c}\log (\mathrm{sSFR}) \\
\left(\mathrm{yr}^{-1}\right)\end{array}$ & $\begin{array}{l}\log (\mathrm{SFR}) \\
\left(M_{\odot} \mathrm{yr}^{-1}\right)\end{array}$ & $\begin{array}{c}\log \left(L_{\mathrm{AGN}}\right) \\
\left(L_{\odot}\right)\end{array}$ & $\begin{array}{c}\log \left(L_{\text {dust }}\right) \\
\left(L_{\odot}\right)\end{array}$ & $\begin{array}{c}\log (\text { Stellar Mass }) \\
\left(M_{\odot}\right)\end{array}$ & $\begin{array}{c}\log \left(M_{\text {dust }}\right) \\
\left(M_{\odot}\right)\end{array}$ & $\begin{array}{l}\text { Cold Dust } \\
\text { Temp. (K) }\end{array}$ & $\begin{array}{l}\text { Warm Dust } \\
\text { Temp. (K) }\end{array}$ \\
\hline $\mathrm{AGN}+\mathrm{SF}^{\mathrm{a}}$ & 1.93 & -11.13 & $-1.34^{\mathrm{f}}$ & 7.70 & - & 9.79 & 6.62 & 16.34 & $-{ }^{a}$ \\
\hline MAGPHYS $^{\mathrm{b}}$ & $0.34^{\mathrm{c}}$ & -11.47 & -1.43 & - & 8.63 & 10.07 & 6.09 & 17.2 & 59.4 \\
\hline Lanz et al. $(2013)^{\mathrm{d}}$ & 0.91 & $-11.67^{\mathrm{e}}$ & -1.35 & & 9.14 & 10.36 & 6.00 & 24.1 & 54.4 \\
\hline
\end{tabular}

Notes.

${ }^{a}$ From Sajina et al. (2006). The model does not assume a specific temperature for the warm dust component.

${ }^{\mathrm{b}}$ Differs from Lanz et al. (2013) by the addition of GALEX and $16 \mu \mathrm{m}$ IRS peak-up point.

${ }^{\mathrm{c}}$ We assume $A_{v}=1.086 \times \tau_{v}$ and find $\mathrm{A}_{v}=0.37$.

d Also uses MAGPHYS with a subset of the points used above.

e Correcting a transcription error in Lanz et al. (2013) for sSFR.

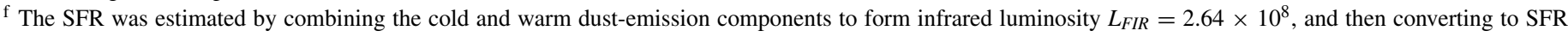
assuming $\log _{10}(\mathrm{SFR})=\log _{10}\left(\mathrm{~L}_{\mathrm{FIR}}\right)-9.762$.

emission to dust heated in "birth clouds." Given that this galaxy contains an AGN, we think it more likely that the AGN rather than birth clouds are responsible for the mid-IR power that the SED modeling seems to require.

\subsection{Star Formation Estimates and Other Properties Based on SED Modeling}

The Sajina et al. (2012) modeling described above provides several important parameters that describe the host galaxy, including the star formation rate, which is given in Table 5. The star formation rate obtained is found to be $0.046( \pm 0.005) M_{\odot} \mathrm{yr}^{-1}$. However, in order to provide an alternative estimate, we also repeat the MAGPHYS modeling performed by Lanz et al. (2013), but using more points. MAGPHYS constrains a range of physical properties in the galaxy, such as far-IR luminosity, star formation rate, total dust mass, and metallicity. This approach has been used successfully in a number of samples, including large SDSS galaxy samples (da Cunha et al. 2010a), ULIRGs (da Cunha et al. 2010b), and in a large study of early- and latetype galaxies in HCGs by Bitsakis et al. (2011). We present the results of the fitted parameters in Table 5, along with those measured by Lanz et al. (2013). Since our values differ only slightly from their fit, we do not present the SED graphically, rather we refer the reader to that paper. Using this method, we find a total star formation rate of $0.038( \pm 0.0002) M_{\odot}$ $\mathrm{yr}^{-1}$. These values are similar to those found using the Sajina et al. model.
The total stellar mass computed by both the Sajina et al. and MAGPHYS models are close to $10^{10} M_{\odot}$, allowing us to calculate the specific star formation rate in the range -11.13 $<$ (sSFR) $<-11.47 \mathrm{yr}^{-1}$ from both methods. This places it squarely between spiral and elliptical galaxies in the Hickson compact group sample of Bitsakis et al. (2010, 2011), consistent with its green valley "transitional" $\mathrm{NUV}-r=4.35$ color $(4.86$ before extinction correction).

A total dust mass was also derived from the MAGPHYS model as $1.24 \times 10^{6} M_{\odot}$. To confirm that this mass is reasonable, we also estimate the dust mass using the formulae of both Magdis et al. (2012, 2013) as well as Dunne et al. (2011). Assuming $T 15 \mathrm{~K}$ and $\beta=1.5$, we obtain $1.67 \times 10^{6} M_{\odot}$ and $1.64 \times 10^{6} M_{\odot}$, respectively. Changing the assumed value of $\beta$ from 1.5 to 2 , reduces the dust mass by $5 \%$. These values are within $25-30 \%$ of the MAGPHYS results and provide some measure of the uncertainty inherent in the different assumptions used to estimate the dust mass.

If the dust mass measured by these methods is accurate, then we can estimate the total gas mass for an assumed dust to gas (DGR) ratio. As shown by Sandstrom et al. (2013), the DGR is strongly dependent on metallicity. If we assume DGR of 0.16 , a value appropriate for Milky Way metallicity galaxies, this would predict $8 \times 10^{7} M_{\odot}$ of gas in the system, more than four times the measured upper limit to the molecular gas from the IRAM CO observations $\left(<2 \times 10^{7} M_{\odot}\right)$. On the other hand, it is well known that the conversion of $I(\mathrm{CO})$ to $N\left(\mathrm{H}_{2}\right), \mathrm{X}_{C O}$, need not 
be constant throughout the universe. One possibility is that $\mathrm{X}_{C O}$ is higher than that assumed, perhaps because the gas acquired by NGC 3226 was of lower metallicity. There is some evidence that the $\mathrm{X}_{C O}$ factor rises rapidly in gas with metallicities below one-third to one-half solar (see Bolatto et al. 2013).

\section{THE RECENT EVOLUTION OF NGC 3226}

NGC 3226 is far from a normal elliptical galaxy. Although there is evidence that it is strongly interacting with NGC 3227 , the complex web of optical and H I gas in the Arp 94 system as a whole suggests that this is not a simple two-body interaction. The optical ripples around both galaxies (see Figure 1) and the complex loops and optical spurs around NGC 3226 may suggest that this galaxy itself is the result of a recent merger, the tidal debris of which may have become shared with NGC 3227. We concentrate here only on the potential connection between northern $\mathrm{H}_{\mathrm{I}}$ plume and recent star formation in and around NGC 3226, as a complete understanding of the many complexities of Arp 94 is beyond the scope of this paper.

There are several possibilities for the origin of the IRACdetected filament. The first is that it is a structure tidally spun out from NGC 3226 from some pre-existing ring of material orbiting the galaxy-perhaps originally captured from the gasrich companion at an earlier time or, more likely, it is the result of late-stage accretion onto NGC 3226 from the large neutral hydrogen plume. We favor the second explanation on kinematic grounds and because material falling back onto both galaxies is expected in mature tidal systems like Arp 94 from modeling arguments (Struck 1997).

Asymmetric strong dust absorption structures, such as the incomplete dust absorption ring structure is seen in the deep HST B-band image of NGC 3226 and reproduced in Figure 12, have often been associated with possible infall events (Tran et al. 2001; Martel et al. 2004). We superimpose the warm $\mathrm{H}_{2}$ distribution from the IRS cube as well as a schematic of the position of the IRAC filament on the image. One possibility is that the dusty/PAH emitting filament (which is associated with the $\mathrm{HI}$ plume) is feeding the warm $\mathrm{H}_{2}$ disk and dust lanes seen in Figure 12. As discussed by (Simões Lopes et al. 2007), the presence of nuclear dust lanes may also correlate with AGN activity.

Is there a kinematic connection that might link the structures? We note that the systemic velocity of NGC 3226 (determined optically) is $1313( \pm 13) \mathrm{km} \mathrm{s}^{-1}$, approximately $100 \mathrm{~km} \mathrm{~s}^{-1}$ higher than the central velocity of the IRAC filament (measured using HI). Atlas ${ }^{3 \mathrm{D}}$ IFU data (Krajnović et al. 2011) provides information on the rotation of the stars $\left(91 \mathrm{~km} \mathrm{~s}^{-1}\right.$ peakvalues along a kinematic major axis P.A. 28deg). The scale of this rotating stellar core is large enough to extend towards the point where the IR filament reaches the minor axis. More recently, unpublished Atlas ${ }^{3 \mathrm{D}}$ imaging of the ionized gas in NGC 3226 (M. Sarzi 2013, private communication) shows a clear connection between a rotating ionized disk in NGC 3226 and the filament-with ionized gas in the filament having a similar velocity to the H I (around $1260-1280 \mathrm{~km} \mathrm{~s}^{-1}$ ). It is not unreasonable to speculate that the $\mathrm{H}$ //dust filament may be feeding both the warm $\mathrm{H}_{2}$ disk and the ionized disk.

As Davis et al. (2011) have pointed out, in fast rotators like NGC 3226, there are many cases where aligned (or almost aligned) stellar and ionized gas disks exist in galaxies that have clear evidence of external accretion. In this case, the kinematic axes of the stellar and ionized-gas disks are not strongly divergent, with position angles of $28^{\circ}$ and $36^{\circ}$, respectively, (see also Krajnović et al. 2011) with an uncertain of $6^{\circ}$. We measure the position angle of the major axis of the incomplete dust ring in Figure 12 to be very similar $\left(28^{\circ}\right)$ to the stellar disk rotation major axis, suggesting that the dust has settled into the rotational plane of the stars. However, we find that the position angle of the warm molecular disk, $45^{\circ}$, is significantly different from the other components, indicating that it may be dynamically unrelaxed.

A rough estimate of the accretion rate onto NGC 3226 can be obtained by dividing the H I mass in the dusty part of the filament by the fallback time onto the galaxy. There is a small velocity gradient along the filament (from inspection of Figure 6 of Mundell et al. 1995), which is $2.9 \mathrm{~km} \mathrm{~s}^{-1} \mathrm{kpc}^{-1}$. Inverting this gives a timescale of approximately $300 \mathrm{Myr}$, which would be an upper limit to the time taken for gas to move along the filament. This timescale could be much shorter since the free-fall time for gas at $10 \mathrm{kpc}$ for a galaxy of mass $10^{11} M_{\odot}$ is approximately 70 Myr. However, given the effects of tidal interactions, gas is unlikely to fall radially onto the galaxy, and so the actual infall rate may be somewhere between these two values. Taking the Hi mass of the inner filament (we only include the gas shown in Figure 7, which is contained within $5 \mathrm{kpc}$ of the galaxy), we obtain a very approximate accretion rate of $d M / d t>0.3 M_{\odot} \mathrm{yr}^{-1}$. As discussed above, this is likely a conservative lower limit because the infall time could shorter.

This rate is close that needed to heat the warm $\mathrm{H}_{2}$ (see Section 4). This accretion rate implies that $10^{8} M_{\odot}$ of $\mathrm{H}_{\text {I }}$ could pile up into the center of NGC 3226 over a few hundred megayears. Hence a substantial disk of material could be formed in the core of the galaxy during the later stages of the collision. We have already shown that the warm molecular hydrogen disk has an $\mathrm{H}_{2}$ mass of $0.75-1.1 \times 10^{7} M_{\odot}$, which could be supplied to the galaxy through from the Hi filament. Since the inner parts of NGC 3226 contain both ionized and warm molecular gas, it is possible that the mainly neutral infalling gas is shock heated as it falls into the nucleus. Attempting to model this possible accretion process through various interstellar mass (ISM) phases is beyond the scope of this observational paper.

\subsection{Is the Measured Star Formation Activity Consistent with the Galaxy Transitioning from Red to Green by Resurgence of Star formation?}

The very low SFR obtained from the UV to far-IR SED fitting, along with the predominantly warm state of the molecular hydrogen in the core, argues against the idea that NGC 3226 could lie in the UV-optical green valley because of a possible resurgence of star formation. For example, a reasonable "extreme" limit for a galaxy to get from a red state to a green valley state is if $5 \%$ of its mass was experiencing a star burst (Schawinski et al. 2009). Smaller ratios of young to old stellar mass would fail completely to move a galaxy away from the (optical) red sequence (Schawinski et al. 2007; Kaviraj et al. 2007). Based on our SED modeling, we estimate the total stellar mass of NGC 3226 to be $1.1 \times 10^{10} M_{\odot}$, and so generating $5 \%$ of this mass with a star formation rate of $0.04 M_{\odot} \mathrm{yr}^{-1}$ would require approximately a Hubble time. Therefore, at its present rate, it is quite implausible that the SFR we presently see could be responsible for the green valley colors of the galaxy. Of course, the SFR of NGC 3226 may have been much larger in the past. In order to significantly perturb normal elliptical colors, at least 


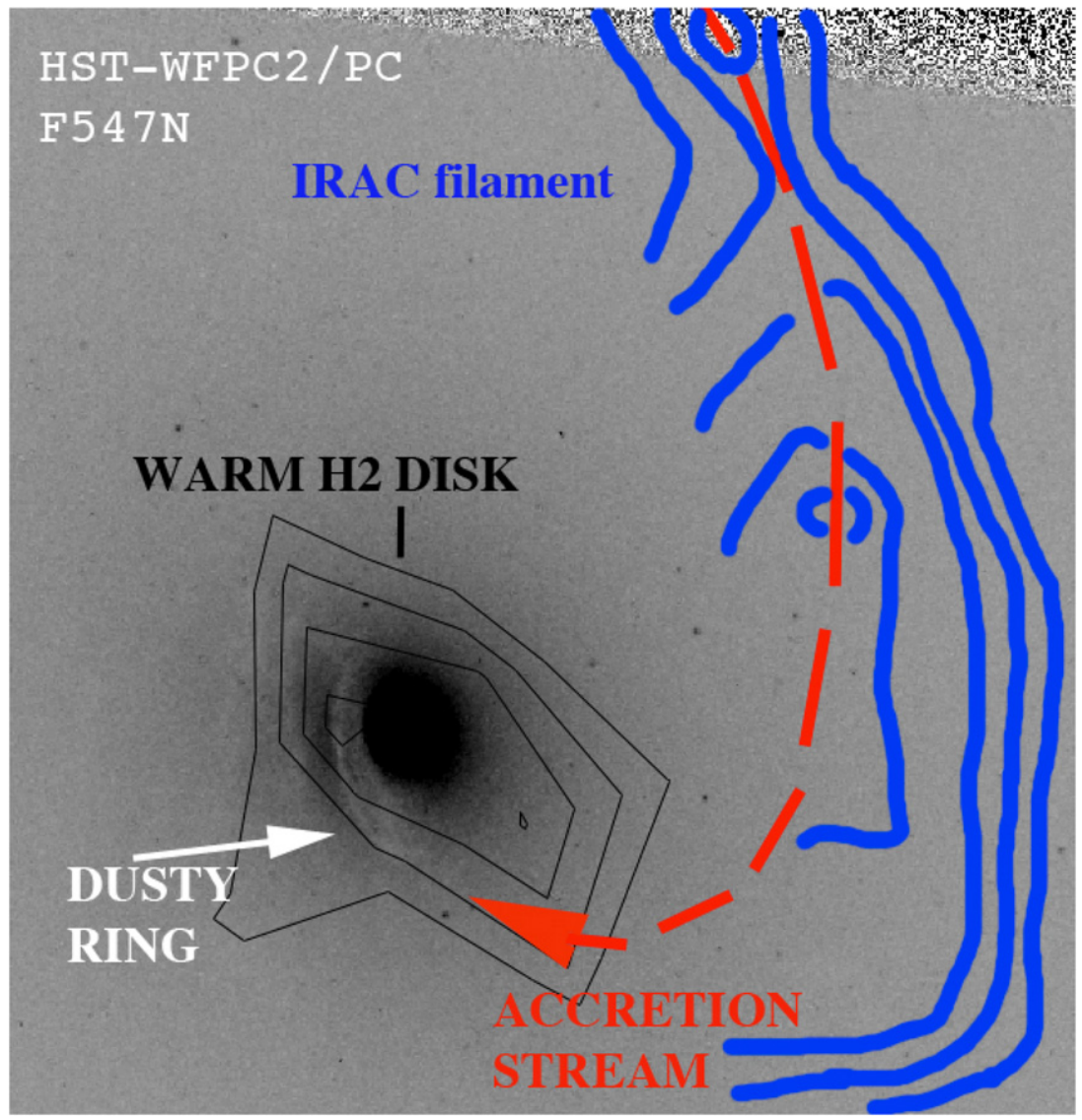

Figure 12. Grayscale image of the core of NGC 3226 showing a partial ring of dust approximately coincident with the warm $\mathrm{H}_{2}$ emission (black contours). The blue contours show the position of the $8 \mu \mathrm{m}$ filament, which lies at the base of the H I plume. The red dotted line is a representation of the possible flow of material from the filament onto the galaxy.

(A color version of this figure is available in the online journal.)

$5 \%-10 \%$ of the mass or $5-10 \times 10^{8} M_{\odot}$ would be required. Even if all the warm $\mathrm{H}_{2}$ that we measure $\left(0.7-1.1 \times 10^{7} M_{\odot}\right)$ plus any possible unobserved cold gas (based on a good upper limit from IRAM) were to turn into stars rapidly, this would not be enough to generate enough blue stars to push the galaxy into the green valley. Thus the green valley nature of NGC 3226 must result from some other cause.

\subsection{Does NGC 3226 Fit into a Broader Picture of Star Formation "Quenched" Galaxies?}

It is tempting to see NGC 3226 as a pathological case that shares little in common with other green valley galaxies. However, the environment of NGC 3226 may share some similarities with denser galaxy environments, like compact groups. This would be especially true if, as we suspect, NGC 3226 is actually itself a relatively recent merger product. The loops and filaments spread throughout the system shown in Figure 2 certainly suggest that the Arp 94 system was composed of more than two galaxies in the past.

Recently, Cluver et al. (2013) have shown that $10 \%$ of HCG galaxies studied by the Spitzer IRS contain galaxies with enhanced $L\left(\mathrm{H}_{2}\right) / L\left(\mathrm{PAH}_{7.7}\right)$ ratios. These MOHEGclassified HCG galaxies predominantly lie in the UV-optical green valley and have unusually low sSFR. NGC 3226 shares similar properties to these galaxies. Johnson et al (2007) and Walker et al. (2010) suggested that these galaxies are undergoing some kind of transition from the blue cloud to the green valley based on their IR colors, and Cluver et al. (2013) suggested that shocks and turbulence may partly be responsible for their green valley colors by helping to suppress star formation. In one example studied in detail, HCG57A, Alatalo et al. (2014a) has shown that star formation seems to be suppressed in regions of the galaxy that are experiencing large disturbances in the COmeasured velocity field caused by a collision with a companion. NGC 3226, which is clearly disturbed, shares some of the same properties of these unusual HCG galaxies.

If NGC 3226 is really a composite galaxy formed by the recent merger of two smaller galaxies, then perhaps the green valley nature of NGC 3226 can be explained as a galaxy caught in the late stages of star formation quenching. For example, the merger may have largely exhausted its gas supply and its ability to form many new stars. Further suppression by the action of shocks from external tidal accretion may also play a role. Some support for this picture can be seen by considering the WISE (Wide-field Infrared Survey Explorer; Wright et al. 2010) colors of the galaxy. Figure 13 shows a WISE color-mass diagram from Alatalo et al. (2014b) showing a strong bifurcation of galaxies into late-type and early-type galaxies from a spectroscopically selected SDSS sample. Alatalo et al. show that there exists an infrared transition zone (IRTZ) that likely contains quenched galaxies, which are moving from the top part of the diagram (blue contours = gas-rich, late-type galaxies) towards the lower part of the diagram (red contours $=$ red cloud galaxies). The IRTZ galaxies are shown to predominantly have the spectral 


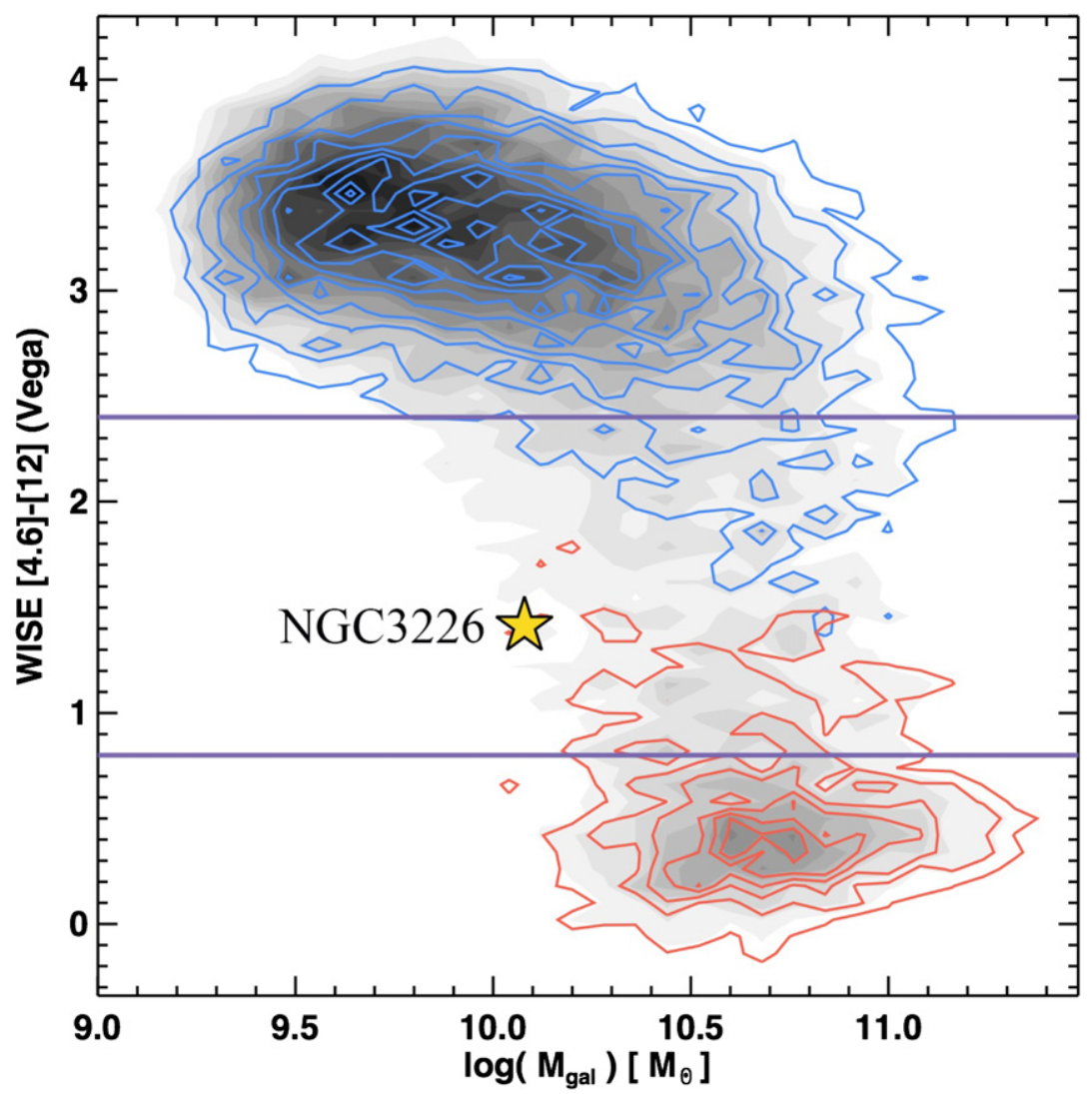

Figure 13. WISE color-mass diagram from Alatalo et al. (2014) based on a sample of spectroscopically selected SDSS galaxies (colored contours), and galaxies from the "Galaxy Zoo" of Schawinski et al. (2014; underlying grayscale). The figure shows the clear bifurcation of early-type (red contours) and late-type (blue contours) galaxies. Contours are 5\% steps of the total galaxy population studied. The area between the two purple horizontal lines in the diagram shows the IRTZ. The galaxies in the IRTZ are shown spectroscopically to be dominated by strong LINERs, a population of Seyferts, and shocked post-starburst galaxy candidates (Alatalo et al. 2014b). NGC 3226 clearly falls in the IRTZ, emphasizing its similarity to a larger body of potentially evolving galaxies (see the text).

(A color version of this figure is available in the online journal.)

signatures of LINER galaxies and shocked post-starburst galaxy candidates based on their optical spectral line ratios. NGC 3226 falls within the lower part of the transition zone. ${ }^{15}$ This is therefore consistent with being part of a larger transitional population of galaxies in which shocks or low-luminosity AGNs play a role in transitioning the galaxies. Further work on the stellar populations in NGC 3226, such as searching for evidence of a post-starburst population hinting at recent quenching, would be worthwhile.

\section{CONCLUSIONS}

By combining mainly archival infrared observations from Spitzer and Herschel, with optical images from the HST, we have expanded our understanding of the green valley elliptical galaxy NGC 3226 and have come to the following conclusions.

1. A narrow filament is detected in the mid-IR, extending into the core of the galaxy, and approximately terminating at the scale of a dusty ring or partial spiral seen in deep optical $H S T$ images and a warm $\mathrm{H}_{2}$ disk (next item). The filament is closely associated with $\mathrm{H} \mathrm{I}$ emission from a much larger H I plume that extends to the north but that kinematically ends at the filament and is spatially coincident with it.

\footnotetext{
15 We obtained the WISE 4.6 and $12 \mu \mathrm{m}$ magnitudes from the ALLWISE survey catalog (Wright et al. 2010). NGC 3226 has w2gmag $(4.6 \mu \mathrm{m})$ and w3 gman $(12 \mu \mathrm{m})$ values of $8.666 \pm 0.008$ and $7.25 \pm 0.02 \mathrm{mag}$.
}

The filament-which mainly glows in the light of PAH molecules and probably molecular hydrogen emission-is likely heated by a string of tiny $\mathrm{H}$ II regions ( $\mathrm{SFR} \sim 3 \times$ $10^{-3} \mathrm{yr}^{-1}$ ), which we detect at the termination of this $\mathrm{H} \mathrm{I}$ plume. The median SFR in the filament clumps is about $7 \%$ of the SFR of the entire galaxy. We suggest that the $\mathrm{H} \mathrm{I}$ plume is feeding material into the center of the galaxy. If correct, these stars forming in the filament will also be added to NGC 3226, creating a new (minor) stellar population.

2. Spitzer IRS observations of NGC 3226 show the existence of a $1 \mathrm{kpc}$ scale warm disk of molecular hydrogen of $0.75-1.1 \times 10^{7} M_{\odot}$, which has a different orientation (by $17^{\circ}$ ) from the major axis of the dusty incomplete nuclear ring and the position angle of the major axis of the fast stellar rotation seen in the galaxy's core. This differences may imply that the warm molecular disk is not yet in dynamical equilibrium with the other components.

3. We can rule out heating of the warm $\mathrm{H}_{2}$ gas disk by either X-rays from the low-luminosity AGN or a dominant PDR component associated with star formation. Instead, we suggest that the $\mathrm{H}_{2}$ line luminosity can be explained by shock heating. We favor $\mathrm{H}$ I gas accretion as the source of the mechanical heating. An accretion rate of $1 M_{\odot} \mathrm{yr}^{-1}$ would be needed (assuming a 10\% efficiency) to balance the observed warm $\mathrm{H}_{2}$ line luminosity, which we show is plausible. 
4. The bulk of the molecular gas in NGC 3226 is warm (with $\left.M\left(\mathrm{H}_{2}\right)_{\text {warm }} / M\left(\mathrm{H}_{2}\right)_{\text {cold }}>0.3\right)$ based on an upper limit to the detection of $\mathrm{CO}$ in the system. Such gas may not be conducive to significant star formation if it is turbulently heated (see Cluver et al. 2010). Alternatively, the conversion factor $\mathrm{X}_{C O}$ assumed for Galactic emission may be a factor of four higher in NGC 3226 if the infalling gas has low metallicity.

5. We measure the SFR in the galaxy globally to be very small $\left(\sim 0.04 M_{\odot} \mathrm{yr}^{-1}\right)$. Since NGC 3226 lies in the $\mathrm{UV}$-optical green valley $\left([\mathrm{NUV}-r]_{\mathrm{corr}}=4.35\right)$, we show that the current SFR in NGC 3226 is insufficient to explain the "green" colors of the galaxy based on a resurgence of star formation unless the SFR was significantly higher in the past. The SED is also consistent with the existence of a hot dust component that we associate with the AGN, which contributes no more than $20 \%$ to the bolometric luminosity of the galaxy.

6. NGC 3226 has some similar properties to a subset of early-type galaxies in Hickson compact groups that may be undergoing rapid evolution (low specific SFR, green $\mathrm{UV}$-optical colors, large warm $\mathrm{H}_{2} / \mathrm{PAH}$ ratio). We also show that NGC 3226 lies in the newly discovered WISE infrared transition zone for SDSS galaxies (Alatalo et al. 2014b). This zone, which may signify evolution from dusty late-type galaxies to dust-free early-type galaxies, has been shown to be dominated by galaxies with LINER and post-starburst, shocked-gas optical spectra. NGC 3226 may, despite its complex dynamical environment, fall on a continuum of galaxies undergoing star formation quenching.

We thank an anonymous referee for very helpful suggestions on how to improve the paper. This work is based, in part, on observations (and archival observations) made with the Spitzer Space Telescope, which is operated by the Jet Propulsion Laboratory, California Institute of Technology under a contract with NASA. The work is also based, in part, on observations made with Herschel, a European Space Agency Cornerstone Mission with significant participation by NASA. Partial support for this work was provided by NASA through an award issued by JPL/Caltech. M.L. acknowledges support from the National Radio Astronomy Observatory (NRAO). NRAO is a facility of the National Science Foundation operated under cooperative agreement by Associated Universities, Inc. T.B. and V.C. acknowledge partial support from EU FP7 grant PIRSESGA-20120-316788. C.G.M. acknowledges support from the Wolfson Foundation, the Royal Society and the Science and Technology Facilities Council. U.L. acknowledges support by the research projects AYA2011-24728 from the Spanish Ministerio de Ciencia y Educación and the Junta de Andalucía (Spain) grants FQM108. P.A. thanks Marc Sarzi and Tim de Zeeuw for information about the ionized gas in NGC 3226 based on unpublished ATLAS ${ }^{3 \mathrm{D}}$ data, and Sergio Fajado-Acosta for advice on the calibration of the IRS peak-up mode.

\section{REFERENCES}

Alatalo, K., Appleton, P. N., Lisenfeld, U., et al. 2014, ApJ, 795, 159 Alatalo, K., Cales, S. L., Appleton, P. N., et al. 2014, ApJL, 794, L13 Appleton, P. N., Guillard, P., Boulanger, F., et al. 2013, ApJ, 777, 66 Appleton, P. N., Xu, K. C., Reach, W., et al. 2006, ApJL, 639, L51 Bitsakis, T., Charmandaris, V., da Cunha, E., et al. 2011, A\&A, 533, A142 Bitsakis, T., Charmandaris, V., Le Floc'h, E., et al. 2010, A\&A, 517, A75
Blanton, M. R., Hogg, D. W., Bahcall, N. A., et al. 2003, ApJ, 594, 186 Bolatto, A. D., Wolfire, M., \& Leroy, A. K. 2013, ARA\&A, 51, 207 Calzetti, D., Kennicutt, R. C., Engelbracht, C. W., et al. 2007, ApJ, 666, 870

Cluver, M. E., Appleton, P. N., Boulanger, F., et al. 2010, ApJ, 710, 248

Cluver, M. E., Appleton, P. N., Ogle, P., et al. 2013, ApJ, 765, 93

da Cunha, E., Charlot, S., \& Elbaz, D. 2008, MNRAS, 388, 1595

da Cunha, E., Charmandaris, V., Díaz-Santos, T., et al. 2010, A\&A, 523, A78 da Cunha, E., Eminian, C., Charlot, S., \& Blaizot, J. 2010, MNRAS, 403, 1894 Davis, T. A., Alatalo, K., Sarzi, M., et al. 2011, MNRAS, 417, 882

de Vaucouleurs, G., de Vaucouleurs, A., Corwin, H. G., Jr., et al. 1991, Third Reference Catalogue of Bright Galaxies (New York: Springer)

Draine, B. T., \& Li, A. 2001, ApJ, 551, 807

Duc, P.-A., Cuillandre, J.-C., Karabal, E., et al. 2014, MNRAS, in press (arXiv:1410.0981)

Dunne, L., Gomez, H. L., da Cunha, E., et al. 2011, MNRAS, 417, 1510

Faber, S. M., Willmer, C. N. A., Wolf, C., et al. 2007, ApJ, 665, 265

Fazio, G. G., Hora, J. L., Allen, L. E., et al. 2004, ApJS, 154, 10

Filho, M. E., Barthel, P. D., \& Ho, L. C. 2006, A\&A, 451, 71

George, I. M., Mushotzky, R. F., Yaqoob, T., et al. 2001, ApJ, 559, 167

Griffin, M. J., Abergel, A., Abreu, A., et al. 2010, A\&A, 518, L3

Guillard, P., Boulanger, F., Pineau Des Forêts, G., \& Appleton, P. N. 2009, A\&A, 502,515

Guillard, P., Boulanger, F., Pineau des Forêts, G., et al. 2012, ApJ, 749, 158

Ho, L. C. 2009, ApJ, 699, 626

Ho, L. C., Filippenko, A. V., \& Sargent, W. L. W. 1997, ApJS, 112, 315

Ho, L. C., Greene, J. E., Filippenko, A. V., \& Sargent, W. L. W. 2009, ApJS, 183,1

Ho, L. C., \& Ulvestad, J. S. 2001, ApJS, 133, 77

Hogg, D. W., Blanton, M. R., Brinchmann, J., et al. 2004, ApJL, 601, L29

Houck, J. R., Roellig, T. L., van Cleve, J., et al. 2004, ApJS, 154, 18

Johnson, K. E., Hibbard, J. E., Gallagher, S. C., et al. 2007, AJ, 134, 1522

Kaviraj, S., Schawinski, K., Devriendt, J. E. G., et al. 2007, ApJS, 173, 619

Kennicutt, R. C., Jr. 1998, ApJ, 498, 541

Kennicutt, R. C., Jr., Hao, C.-N., Calzetti, D., et al. 2009, ApJ, 703, 1672

Krajnović, D., Emsellem, E., Cappellari, M., et al. 2011, MNRAS, 414, 2923

Lacy, M., Sajina, A., Petric, A. O., et al. 2007, ApJL, 669, L61

Lanz, L., Zezas, A., Brassington, N., et al. 2013, ApJ, 768, 90

Lepp, S., \& McCray, R. 1983, ApJ, 269, 560

Lisenfeld, U., Mundell, C. G., Schinnerer, E., Appleton, P. N., \& Allsopp, J. 2008, ApJ, 685, 181

Liu, H., \& Wu, Q. 2013, ApJ, 764, 17

Magdis, G. E., Daddi, E., Béthermin, M., et al. 2012, ApJ, 760, 6

Magdis, G. E., Rigopoulou, D., Helou, G., et al. 2013, A\&A, 558, A136

Makovoz, D., \& Marleau, F. R. 2005, PASP, 117, 1113

Maraston, C. 2005, MNRAS, 362, 799

Martel, A. R., Ford, H. C., Bradley, L. D., et al. 2004, AJ, 128, 2758

Martin, D. C., Wyder, T. K., Schiminovich, D., et al. 2007, ApJS, 173, 342

Mullaney, J. R., Alexander, D. M., Goulding, A. D., \& Hickox, R. C. 2011, MNRAS, 414, 1082

Mundell, C. G., James, P. A., Loiseau, N., Schinnerer, E., \& Forbes, D. A. 2004, ApJ, 614, 648

Mundell, C. G., Pedlar, A., Axon, D. J., Meaburn, J., \& Unger, S. W. 1995, MNRAS, 277, 641

Nagar, N. M., Falcke, H., \& Wilson, A. S. 2005, A\&A, 435, 521

Ogle, P., Boulanger, F., Guillard, P., et al. 2010, ApJ, 724, 1193

Ogle, P. M., Lanz, L., \& Appleton, P. N. 2014, ApJL, 788, L33

Ott, S., Bakker, J., Brumfitt, J., et al. 2006, ASPC, 351, 516

Peterson, B. W., Appleton, P. N., Helou, G., et al. 2012, ApJ, 751, 11

Pilbratt, G. L., Riedinger, J. R., Passvogel, T., et al. 2010, A\&A, 518, L1

Poglitsch, A., Waelkens, C., Geis, N., et al. 2010, A\&A, 518, L2

Rieke, G. H., Young, E. T., Engelbracht, C. W., et al. 2004, ApJS, 154, 25

Rigopoulou, D., Kunze, D., Lutz, D., Genzel, R., \& Moorwood, A. F. M. 2002, A\&A, 389, 374

Roussel, H. 2013, PASP, 125, 1126

Roussel, H., Helou, G., Hollenbach, D. J., et al. 2007, ApJ, 669, 959

Rubin, V. C., \& Ford, W. K. 1968, ApJ, 154, 431

Sajina, A., Scott, D., Dennefeld, M., et al. 2006, MNRAS, 369, 939

Sajina, A., Yan, L., Fadda, D., Dasyra, K., \& Huynh, M. 2012, ApJ, 757, 13

Sandstrom, K. M., Leroy, A. K., Walter, F., et al. 2013, ApJ, 777, 5

Schawinski, K., Kaviraj, S., Khochfar, S., et al. 2007, ApJS, 173, 512

Schawinski, K., Urry, C. M., Simmons, B. D., et al. 2014, MNRAS, 440,889

Schawinski, K., Virani, S., Simmons, B., et al. 2009, ApJL, 692, L19

Schweizer, F., \& Seitzer, P. 1988, ApJ, 328, 88

Simien, F., \& Prugniel, Ph. 2002, A\&A, 384, 371

Simões Lopes, R. D., Storchi-Bergmann, T., de Fátima Saraiva, M., \& Martini, P. 2007, ApJ, 655, 718 
Smith, J. D. T., Armus, L., Dale, D. A., et al. 2007a, PASP, 119, 1133

Smith, J. D. T., Draine, B. T., Dale, D. A., et al. 2007b, ApJ, 656, 770

Strateva, I., Ivezić, Ž., Knapp, G. R., et al. 2001, AJ, 122, 1861

Struck, C. 1997, ApJS, 113, 269

Sturm, E., Bauer, O. H., Brauer, J., et al. 1998, in ASP Conf. Ser. 145 Astronomical Data Analysis Software and Systems VII, ed. R. Albrecht, R. N. Hook, \& H. A. Bushouse (San Francisco, CA: ASP), 161
Tang, Y., Gu, Q.-S., Huang, J.-S., \& Wang, Y.-P. 2009, MNRAS, 397, 1966 Thilker, D. A., Bianchi, L., Schiminovich, D., et al. 2010, ApJL, 714, L171 Tran, H. D., Tsvetanov, Z., Ford, H. C., et al. 2001, AJ, 121, 2928

Walker, L. M., Johnson, K. E., Gallagher, S. C., et al. 2010, AJ, 140, 1254

Westoby, P. B., Mundell, C. G., \& Baldry, I. K. 2007, MNRAS, 382, 1541

Wright, E. L., Eisenhardt, P. R. M., Mainzer, A. K., et al. 2010, AJ, 140, 1868 Young, L. M., Bureau, M., Davis, T. A., et al. 2011, MNRAS, 414, 940 